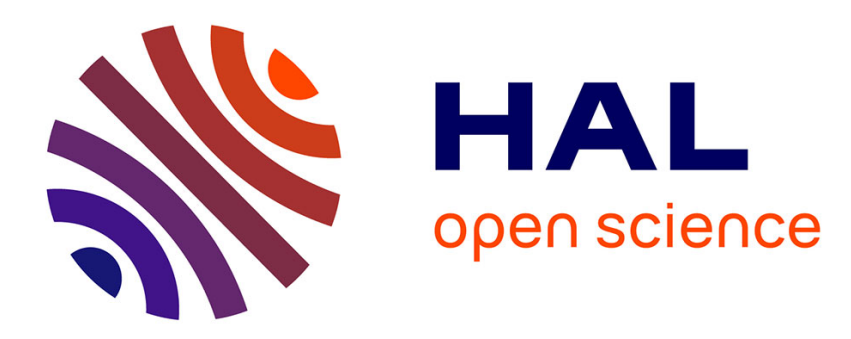

\title{
Qualitative properties of a nonlinear system for laminar flames without ignition temperature
}

\author{
M. Marion
}

\section{To cite this version:}

M. Marion. Qualitative properties of a nonlinear system for laminar flames without ignition temperature. RR-0293, INRIA. 1984. inria-00076264

\section{HAL Id: inria-00076264 https://hal.inria.fr/inria-00076264}

Submitted on 24 May 2006

HAL is a multi-disciplinary open access archive for the deposit and dissemination of scientific research documents, whether they are published or not. The documents may come from teaching and research institutions in France or abroad, or from public or private research centers.
L'archive ouverte pluridisciplinaire HAL, est destinée au dépôt et à la diffusion de documents scientifiques de niveau recherche, publiés ou non, émanant des établissements d'enseignement et de recherche français ou étrangers, des laboratoires publics ou privés. 


\section{Rapports de Recherche}

\section{No 293}

\section{QUALITATIVE PROPERTIES OF A NONLINEAR SYSTEM FOR LAMINAR FLAMES WITHOUT IGNITION TEMPERATURE}

\section{Institut National de Recherche en Informatique et en Automatique}

Domaine de Voluceau Rocquencourt 3.4.

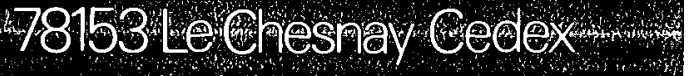
France

Tél.:(3) 9549020 
Qualitative properties of a nonlinear system

for laminar flames without ignition temperature

\title{
Martine MARION *
}

Abstract : We are interested in a system of two nonlinear differential equations $-u^{\prime \prime}+c u^{\prime}=f_{\varepsilon}(u) v,-\lambda v^{\prime \prime}+c v^{\prime}=-f_{\varepsilon}(u) v$ on $\mathbf{R}$, with the boundary conditions: $\mathrm{u}(-\infty)=0, \mathrm{v}(-\infty)=1, \mathrm{u}(+\infty)=1, \mathrm{v}(+\infty)=0$, where the unknowns are the functions $u, v: \mathbb{R} \rightarrow[0,1]$ and the scalar $c$. This system arises in the combustion of laminar plane flames. $f_{\varepsilon}$ is $\oint_{\varepsilon}^{1}$ with $f_{\varepsilon}>0$ on $\left.] 0,1\right]$ and $f_{\varepsilon}(0)=0$. Different qualitative properties of the model are studied here : existence of solutions (we prove that there is a continuum of solutions for $c \geq c_{\min }$ ), asymptotic behaviour of these solutions for an infinite activation energy, relationship with the model of ignition temperature, and lastly convergence of the bounded interval approximation.

\author{
Etude mathêmatique d'un modèle de flamme \\ laminaire sans température d'ignition : II - Cas du système
}

Martine MARION *

Résumé : On s'intéresse à un système de deux èquations différentielles non linéaires $-u^{\prime \prime}+c u^{\prime}=f_{\varepsilon}(u) v,-\lambda v^{\prime \prime}+c v^{\prime}=-f_{\varepsilon}(u) v$ sur $\mathbb{R}$, avec les conditions aux $1 i-$ mites : $\mathrm{u}(-\infty)=0, \mathrm{v}(-\infty)=1, \mathrm{u}(+\infty)=1, \mathrm{v}(+\infty)=0$, où les inconnues sont les deux applications $u, v: \mathbb{R} \rightarrow[0,1]$, et le scalaire $c$. Ces équations interviennent dans la combustion de flammes laminaires planes. $f_{\varepsilon}$ est $C_{1}^{1}$ avec $f_{\varepsilon}>0$ sur $\left.] 0,1\right]$ et $\mathrm{f}_{\varepsilon}(0)=0$. Différentes propriétés qualitatives du modèle sont étudiées ici : existence de solutions (on démontre qu'il existe un continuum de solutions pour $c \geq c_{m i n}$ ), comportement asymptotique de ces solutions pour une énergie d'activation infinie, lien avec le modèle de la température d'ignition, et enfin, convergence de l'approximation sur un intervalle borné.

INRIA, Domaine de Voluceau, Rocquencourt, B.P. 105, 78153 Le Chesnay Cedex. 


\section{INTRODUCTION.}

One of the simplest problems of combustion is the planar premixed flame, that is the one-dimensional deflagration wave. In a case of a single-step reaction involving one reactant, it reduces to the following system of two reaction-diffusion equations (see Buckmaster and Ludford [2], Williams [10]), where the unknowns are the functions $u, v: \mathbb{R} \rightarrow[0,1]$ and the scalar $c \in \mathbb{R}:$

(0.1) $\left\{\begin{array}{l}-u^{\prime \prime}+c u^{\prime}=f(u) v, \\ -\lambda v^{\prime \prime}+c v^{\prime}=-f(u) v, \\ u(-\infty)=0, \quad u(+\infty)=1, \\ v(-\infty)=1, \quad v(+\infty)=0 .\end{array}\right.$

$u$ is the (reduced) temperature of the mixture, $v$ is the (reduced) concentration of the reactant and $c$ is an (unknown) massflux. The parameter $\lambda>0$ is the inverse of the Lewis number. $f$ is a renormalized reaction term depending on a parameter $\varepsilon>0$ which is the inverse of the activation energy.

The exact term $f$ arising from Arrhenius Law satisfies $f>0$ on $[0,1]$; in particular, since $f(0)>0$, it is easily seen that (0.1) has no solution; this is the classical "cold boundary difficulty" well known to the chemists. Therefore, one modifies the reaction term. A first approach is to introduce an ignition temperature $\theta$ : one assumes that there exists some $\theta \in] 0,1[$ such that

$(0.2) f \equiv 0$ on $[0, \theta)$ and $f>0$ on $(\theta, 1]$.

Another way is to alter the reaction term so that $f$ satisfies :

$(0.3) \mathrm{f}>0$ on $] 0,1]$ and $f(0)=0$.

Our purpose here is the study of this latter model. The first model has been considered in many works and more recently by Berestycki, Nicolaenko and Scheurer (see [1], and the references given theiren). Under condition (0.2), with f Lipschitz continuous except possibly at $\theta$, these authors proved, by a topological degree argument, that (0.1) has a solution (whose uniqueness they conjectured); moreover, they derived the asymptotic behaviour of this solution, as $\varepsilon \rightarrow 0$.

The second model was only studied rigorously in the case $\lambda=1$, which is very particular, because it reduces to a scalar equation (indeed, one then has $v=1-u$ ). For this 
equation, Fife [4.], Johnson [5], Uchiyama [9] showed that there exists a continuum of solutions (for $c \geq c_{0}>0$ ). Their approach relies on a phase plane argument. In [7], we derived the same result by a shooting method, and we obtained many further qualitative properties ; in pa:ticular, we investigated the asymptotic behaviour of the different solutions, as $\varepsilon \rightarrow 0$.

The present paper is a sequel of [7] and deals with the model with no ignition temperature in the case of any Lewis number (that is any $\lambda>0$ ). Following our study of the scalar case in [i], we consider the model from a triple point of view. - Determination of the set of the solutions. - Asymptotic analysis of these solutions for an infinite activation energy (this type of asymptotics is very much used in practice; see $[2,10])$. - Relationship with the model with ignition temperature. Furthermore, in view of numerical calculations, we investigate the approximation of Problem $(0.1)$ by an analogous one on a bounded interval $I_{a}=[-a,+a]$.

For a technical reason (monotony lemma), we obtain much more detailed answers in the case $\lambda<1$. The paper is divided into two parts : in section 1 , we consider the general case $\lambda>0$; then, Section 2 is devoted to the case $\lambda<1$. More precisely, the plan of the paper is as follows :

0 . Introduction.

1. General results.

1.1 The solutions of $(0.1)$.

1.2 The role of the ignition temperature.

1.2.1 Construction of a solution of $(0.1)$.

1.2.2 Asymptotic analysis.

1.3 The problem on a bounded interval.

3. Case of the Lewis number larger than 1.

2.1 Monotony lemma.

2.2 Applications.

2.2.1 Determination of the set of solutions of $(0.1)$.

2.2.2 Relationship with the model with ignition temperature.

2.3 Further remarks on the asymptotic analysis. 
While the proofs for the scalar case ([7]) are elementary, the study of the system asks for more elaborated techniques, namely, here, the topological degree and the (local) theory of dynamical systems.

The principal open questions are the asymptotic analysis (essentially the determination of $\lim _{\varepsilon \rightarrow 0} c_{0, \varepsilon}$, see below) and the case $\lambda>1$.

\section{Main results.}

We assume that $\mathbf{f}$ satisfies :

(0.4) $\{\mathrm{f}$ is Lipschitz continuous on $[0,1]$, $[\mathrm{f}>0$ on $] 0,1]$. and $\mathrm{f}(0)=0$.

The main existence results that we obtain (Theorems 1.1 and 2.7 ) can be summarized in the following :

Theorem 0.1 : Under condition (0.4):

(a) Let any $\lambda>0$ be given. There exist two constants $0<\underline{c} \leq \bar{c}$ such that $\forall c \leq \underline{c}$ $(0.1)$ has no solution of the form $(u, v, c)$ and $\forall c \geq \vec{c}(0.1)$ has a solution of the form $(u, v, c)$. Moreover, under the additional condition that $f$ is of class $C$ ! in a neighbourhood of $1, \forall c>0$, there exists at most a couple (u,v) lup to a translation of the origin) such that $(u, v, c)$ is a solution of $(0.1)$.

(b) Suppose that 6 is of class 1 in a neighbourhood of 1 and let $0<\lambda<1$. Then there exists $c_{0}>0$ such that $(0.1)$ has a solution of the form $(u, v, c) i f$ and only if $c \geq c_{0}$.

The methods that we employ here enable us to show a new uniqueness result relative to the model with ignition temperature. Namely, we derive :

Theorem 0.2 : Suppose that condition(0.2) holds with 6 Lipschitz continuous on $[0,1]$, except possibly at $\theta$, and of class $f^{1}$ in a neighbourhood of 1 . For any $0<\lambda<1$, (0.1) has a unique solution (up to a translation of the origin).

Hence, as it is the case in the scalar equation, the existence results for the two models are quite different. 
Next, we consider the relationship between the solutions of the two models. Let f satisfy $(0.4)$ and, for any $\theta \in] 0,1[$, let us introduce the following problem :

(0.5) $\left\{\begin{array}{l}-u^{\prime \prime}+c u^{\prime}=f(u) x_{[\theta, 1]}^{(u) v,} \\ -\lambda v^{\prime \prime}+c v^{\prime}=-f(u) x_{[\theta, 1]}(u) v, \\ u(-\infty)=0, \quad u(0)=z_{0}, \quad u(+\infty)=1, \\ v(-\infty)=1, \quad v(+\infty)=0,\end{array}\right.$

where $X_{[\theta, 1]}$ denotes the characteristic function of $[\theta, 1]$ and $z_{0}$ is some fixed real $\epsilon] 0,1\left[\right.$ (the condition $u(0)=z_{0}$ is a normalization related to the translational invariance). From [1], we know that, for any $\lambda>0,(0.5)$ has a solution $\left(u_{\theta}, v_{\theta}, c_{\theta}\right)$; in the case $\lambda<1$, this solution is unique (Theorem 0.2 above). We will show here that there exists a sequence $\left(\theta_{n}\right)$ with $\lim \theta_{n}=0$ such that $\left(u_{\theta_{n}}, v_{\theta_{n}}, c_{\theta_{n}}\right)$ converges to a solution of $(0.1)$; moreover, in the case $\lambda<1$, the whole family $\left(u_{\theta}, v_{\theta}, c_{\theta}\right)$ converges, as $\theta \rightarrow 0$, and the limit is the solution $\left(u_{0}, v_{0}, c_{0}\right)$ of $(0.1)$ corresponding to the lower $c, c_{0}$, defined at Theorem 0.1 (b).

Lastly, we consider the question of large activation energy asymptotics. We assume that $\mathrm{f}=f_{\varepsilon}$ behaves like $\mathrm{f}_{\varepsilon}(\mathrm{x})=\frac{1}{\varepsilon^{2}} \exp \left(\frac{1}{\varepsilon} \frac{\mathrm{x}-1}{\mathrm{x}}\right)$ (see Section 1.2.2. for the precise conditions; let us recall that $\varepsilon$ is the inverse of the activation energy). In general, we only determine the asymptotic behaviour as $\varepsilon \rightarrow 0$ of the particular solution obtained above from the limiting model with ignition temperature. Indeed, for any $\varepsilon>0$, let us denote by $\left(u_{o, \varepsilon}, v_{o, \varepsilon}, c_{o, \varepsilon}\right)$ this solution. We show that $c_{0, \varepsilon}$ is bounded independently of $\varepsilon>0$; moreover, if $\left(\varepsilon_{n}\right)$ is a sequence with lim $\varepsilon_{n}=0$ and $\lim c_{0, \varepsilon_{n}}=c_{o}$, then $\left(u_{0, \varepsilon_{n}}, v_{o, \varepsilon_{n}}\right)$ converges to the unique solution $\left(u_{0}, v_{0}\right)$ of the following system :

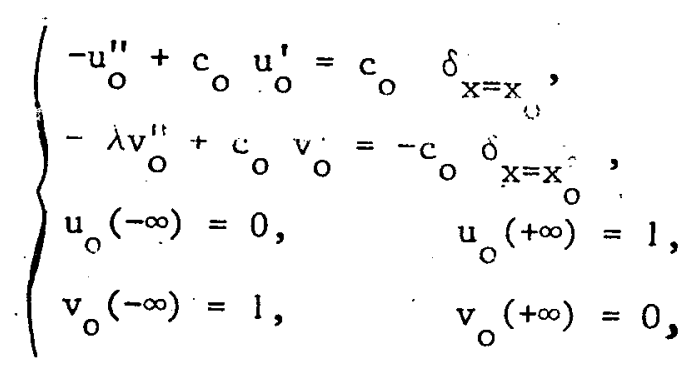


where $x_{0}$ is uniquely determined by the condition $u_{0}(0)=z_{0}$. One of the main steps in the proof of this result is to remark that the function $h_{o, \varepsilon}=u_{o, \varepsilon}^{\prime}\left(u_{o, \varepsilon}^{-l}\right)$ is differen-
tiable at 0 and $h_{o, \varepsilon}^{\prime}(0)=0$.

In the case $\lambda<1$, we obtain informations on the asymptotic behaviour of all the other solutions of $(0.1)$; if $c$ is large enough, the corresponding solution of (0.1) (defined for $\varepsilon \leq \varepsilon_{0}$ ) or, at least, a subsequence converges as $\varepsilon \rightarrow 0$ to a couple (u,v) which does not satisfy any more the boundary conditions at $-\infty$. This illustrates quite well the cold boundary difficulty. The absence of any ignition temperature leads to infinitely many solutions. But, here, we show rigorously that, at the limit $\varepsilon=0$, only one solution satisfies the correct boundary conditions. 


\section{1 - GENERAL RESULTS}

This section is devoted to the study of the laminar plane flame for any Lewis number. Section 1.1 deals with existence results. In Section 1.2, we investigate the relationship between the considered model and the one with ignition temperature and we derive the asymptotic behaviour for large activation energy of a particular solution of (0.1). In Section $1.9 \ldots$ sisily lise pr.iblem analogous to (0.1) on a_bounded interval $[-a,+a]$ and the convergence of solutions as $a^{-} \rightarrow+\infty$. Through out this section, we assume condition $(0.4)$ and we denote :

(1.1) $\quad \sigma=\sup _{x \in] 0,1[} \frac{f(x)(1-x)}{x}$,

(1.2) $\left\{\begin{array}{l}\Lambda=\max \left(\frac{1}{\lambda+|\lambda-1|}, \frac{1}{1+|\lambda-1|}\right), \\ \Lambda^{\prime}=\min \left(\frac{\lambda+|\lambda-1|}{\lambda}, \frac{|\lambda-1|+1}{\lambda}\right) .\end{array}\right.$

1.1. The solutions of $(0.1)$.

Our main results can be summarized in the following :

Theorem 1.1 : Under condition (0.4):

(i) $\forall c \leq \sqrt{2 \Lambda \int_{0}^{1} f(s)(1-s) d s},(0.1)$ has no solution of the form $(u, v, c)$.

(ii) $\left.\forall z_{0} \epsilon\right] 0,1\left[, \quad \forall c \geq 2 \sqrt{\sigma \Lambda^{\prime}},(0.1)\right.$ has got a solution $(u, v, c)$

satisfying $u(0)=z_{0}$.

(ii) If we assume, in addition, that $f$ is of class $C^{1}$ in a neighbourhood of 1 , then, $\forall c>0$, there exists at most one solution of the form $(u, v, c)$ lup to a translation of the origin).

The proof of this theorem will be given in three steps. 
a) Preliminary results; proof of (i).

Let us extend $f$ to $\mathbb{R}$ by setting; :

$f(x)=0 \quad \forall x \leq 0, \quad f(x)=f(1) \quad \forall x \geq 1$

The first lemma states a classical and very useful result; let us assume that, for some $c>0$, there exist $\left.x_{0}, x_{1} \in j^{-}=\infty,+\infty\right]$ and two functions $u, v \in C^{2}$ $\left(\left[x_{0}, x_{1}[, \mathbb{R})\right.\right.$ satisfying the following conditions :

(1.3) $-\mathrm{u}^{\prime \prime}+\mathrm{cu} \mathrm{u}^{\prime}=\mathrm{f}(\mathrm{u}) \mathrm{v}$ and $-\lambda \mathrm{v}^{\prime \prime}+\mathrm{cv}{ }^{\prime}=-\mathrm{f}(\mathrm{u}) \mathrm{v}$ on $\left[\mathrm{x}_{0}, \mathrm{x}_{1}[\right.$.

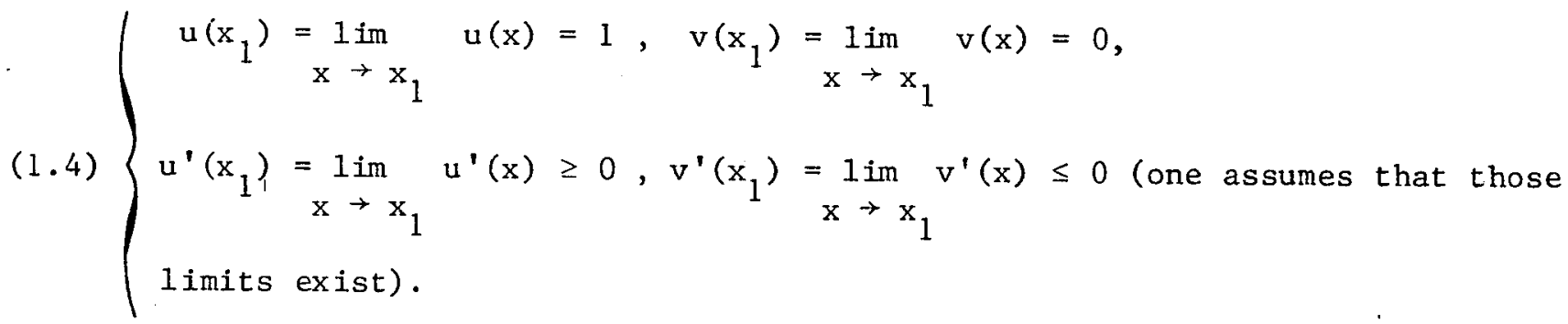

(1.5) $\quad \mathrm{v} \geq 0$ on $] \mathrm{x}_{0}, \mathrm{x}_{1}[$.

Lemma 1.2 : Under conditions (1.3), (1.4) and (1.5), we have:

(i) $\mathrm{u}^{\prime} \geq 0$ and $\mathrm{v}^{\prime} \leq 0$ on $\left[\mathrm{x}_{0}, \mathrm{x}_{1}[\right.$.

(ii) Moreover, under the additional assumption :

(1.6) There exists $x_{2} \in\left[x_{0}, x_{1}\right]$ such that $-u^{\prime}\left(x_{2}\right)+c u\left(x_{2}\right)-\lambda \cdot v^{\prime}\left(x_{2}\right)+c \cdot v\left(x_{2}\right)=c$ we have, $\forall x \in\left[x_{0}, x_{1}\right]$,

(1.7) $\begin{cases}\mid \mathrm{u} & (\mathrm{x})+\mathrm{v}(\mathrm{x})-1|\leq| \lambda-1 \mid \mathrm{v}(\mathrm{x}) \\ \mid \mathrm{u} & (\mathrm{x})+\mathrm{v}(\mathrm{x})-1 \mid \leq \frac{|\lambda-1|}{\lambda}(1-\mathrm{u}(\mathrm{x}))\end{cases}$

(1.8) $\left\{\begin{array}{l}|u(x)+\lambda v(x)-1| \leq|\lambda-1| v(x) \\ |u(x)+\lambda v(x)-1| \leq|\lambda-1|(1-u(x))\end{array}\right.$ 
The proof, wich is easy, will be omitted (see for example Berestycki, Nicolaenko and Scheurer [1]).

Corollary 1.3 : Under conditions (1.3), (1.4), (1.5) and (1.6), we have

(1.9) $\forall x \in\left[x_{0}, x_{1}\right] \quad \Lambda(1-u(x)) \leq v(x) \leq \Lambda^{\prime}(1-u(x))$

Lemma 1.4 : Suppose $\mathrm{u}, \mathrm{v}, \mathrm{c}$ is a solution of (0.1). Then :

$$
c>0, \quad 0<\mathrm{u}^{\prime} \leq \mathrm{c}, \quad \frac{-\mathrm{c}}{\lambda} \leq \mathrm{v}^{\prime}<0,
$$

$\mathrm{u}$ and $\mathrm{v}$ satisfy the inequalities (1.7), (1.8) and (1.9)

Proof : Let $a<b$ and let $u$ s integrate the equation for $u$ on $[a, b]$ to obtain the relation :

$$
-u^{\prime}(b)+u^{\prime}(a)+c(u(b)-u(a))=\int_{a}^{b} f(u) v d x
$$

From (1.10), it is easily seen that :

$$
\lim _{x \rightarrow \pm \infty} u^{\prime}(x)=0 \quad \text { and } \quad c=\int_{-\infty}^{+\infty} f(u) v d x>0
$$

So we can apply lemma 1.2 (and Corollary 1.3 ) to $(u, v)$.

Therefore it remains to show the inequalities : $0<u^{\prime} \leq c$ and $-\frac{c}{\lambda}<v^{\prime} \leq 0$.

Suppose that $\exists x_{0} \in \mathbb{R}$ with $u^{\prime}\left(x_{0}\right)=0$. If $f\left(u\left(x_{0}\right)\right) v\left(x_{0}\right)>0$, from the equation satisfied by $u$, we have $u^{\prime \prime}\left(x_{0}\right)<0$, which is impossible (Lemma 1.2). On the other hand, if $f\left(u\left(x_{0}\right)\right) v\left(x_{0}\right)=0$, then $u\left(x_{0}\right)=0$ or $v\left(x_{0}\right)=0$, hence $u \equiv 0$ or $v \equiv 0$ which is again a contradiction.

Last1y $e^{-c x} u^{\prime}(x)=\int_{x}^{+\infty} e^{-c s} f(u(s)) v(s) d s \leq e^{-c x} \int_{x}^{+\infty} f(u) v d s=e^{-c x} c$

Thus we have proved that $0<u^{\prime} \leq c$. In the same way, it can be shown that $-\frac{c}{\lambda} \leq v^{\prime}<0$.

Lemma 1.5: Suppose u, v, $\mathrm{c}$ is a solution of $(0.1)$. Then :

$$
c>\sqrt{2 \Lambda \int_{0}^{1} f(s)(1-s) d s}
$$


Proof : Multiplying the equation for u successively iby $u$ and $u^{\prime}$ and integrating over $[a, b] \subset \mathbb{R}$, we obtain :

$$
\begin{aligned}
& \text { (1.11) }-u(b) u^{\prime}(b)+u(a) u^{\prime}(a)+\int_{a}^{b} u^{\prime 2} d x+\frac{c}{2}\left(u^{2}(b)-u^{2}(a)\right)=\int_{a}^{b} f(u) v u d x \\
& (1.12)-\frac{1}{2} u^{\prime 2}(b)+\frac{1}{2} u^{\prime 2}(a)+c \int_{a}^{b} u^{\prime 2} d x=\int_{\eta}^{b} f(u) v u^{\prime} d x
\end{aligned}
$$

We can let $a \rightarrow-\infty$ and $b \rightarrow+\infty$ in $(1.10),(1.11$ ) ano $(i .1 /)$.

Since $\int_{-\infty}^{+\infty} f(u) v u d x<\int_{-\infty}^{+\infty} f(u) v d x,(1.10)$ and (1.11) tmply :

$$
\int_{-\infty}^{+\infty} u^{\prime 2} d x<\frac{c}{2}
$$

Thus combining this last inequality with (1.2) and using the fact that $c>0$ (lemma 1.4), we deduce

$$
\int_{-\infty}^{+\infty} f(u) v u^{\prime} d x<\frac{c^{2}}{2}
$$

It is now easy to conclude the proof, as $v \geq \Lambda(1-u)$ and $u^{\prime}>0$.

\section{(b) Proof of Theorem 1.1 (ii)}

The first (and main) step will consist in proving the following Proposition :

Proposition 1.6: $\left.\forall z_{0} \in\right] 0,1\left[\right.$ and $\forall c>0$, there exist $u, v \in C_{c}^{2}(\mathbb{R}, \mathbb{R})$ such that :

(1.13) $\left\{\begin{array}{l}-u^{\prime \prime}+c u^{\prime}=f(u) v \text { and }-\lambda v^{\prime \prime}+c v^{\prime}=-f(u) v \text { on } \mathbb{R}, \\ u(0)=z_{0}, u(+\infty)=1, v(+\infty)=0, \\ u^{\prime}>0 \text { and } v^{\prime}<0 \text { on } \mathbb{R}, \\ u \text { and } v \text { satis } j y \text { the relations (1.7), (1.8) and (1.9). }\end{array}\right.$ 
We will then show that, if $c$ is large enough, the couple defined by Proposition 1.6 , satisfies $u(-\infty)=0$ and $v(-\infty)=1$ and hence is a solution of $(0.1)$. This will complete the proof of Theorem 1.1 (ii).

Let $\left.z_{0} \in\right] 0,1[$ and $c>0$. The couple $(u, v)$ of Proposition 1.6 will be obtained as the limit as $a \rightarrow+\infty$ of the solutions of an auxiliary'problem posed on a bounded interval $[0, a]$. More precisely, $\forall a>0$, let us consider the following problem : $(1.14)\left\{\begin{array}{l}\text { To find } u, v \in \mathscr{\varphi}_{,}^{2}([0, a], \mathbb{R}) \text { and } \mu \geq 0 \text { such that : } \\ -u^{\prime \prime}+c u^{\prime}=f(u) v \text { and }-\lambda v^{\prime \prime}+c v^{\prime}=-f(u) v \text { on }[0, a] \\ u(0)=z_{0}, u(a)=1, \quad u^{\prime}(0)=\mu, \\ -\lambda v^{\prime}(0)+c v(0)=c\left(1-z_{0}\right)+\mu, \quad v(a)=0\end{array}\right.$

Our purpose is to show that, $¥ a>0,(1.14)$ has got a solution $\left(u_{a}, v_{a}, \mu_{a}\right)$ and that $\left(u_{a}, v_{a}\right)$ converges, as $a \rightarrow+\infty$, to $(u, v)$ satisfying the conclusions of proposition 1.6 .

We solve (1.14) by using a topological degree argument. More precisely, $\forall \tau \in[0,1]$ let us denote by (1.15) the problem analogous to $(1.14)$, where $f$ is repla-

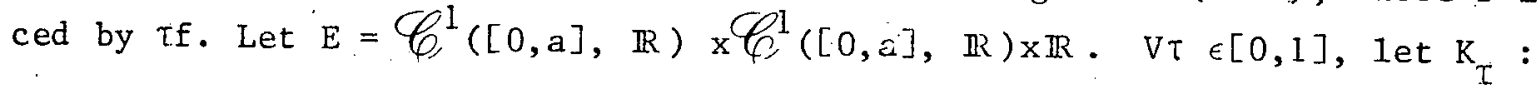
$E \rightarrow E$ be defined by :

$$
\mathrm{K}_{\tau}(\mathrm{u}, \mathrm{v}, \mu)=(U(\mathrm{u}, \mathrm{v}, \mu), \mathrm{v}(\mathrm{u}, \mathrm{v}, \mu), \mu-\mathrm{u}(\mathrm{a})+1)
$$

where $(U, V)$ is the unique solution of the following linear system :

$$
\left\{\begin{array}{l}
-U^{\prime \prime}+c U^{\prime}=\tau f(u) v, \quad-\lambda V^{\prime \prime}+c V^{\prime}=-\tau f(u) v, \\
U\left((0)=z_{0}, \quad U^{\prime}(0)=\mu,\right. \\
-\lambda V^{\prime}(0)+c V(0)=c\left(1-z_{0}\right)+\mu, \quad V(a)=0 .
\end{array}\right.
$$

Then the solutions of (1.15) are precisely the fixed points of $k_{\tau}$ in $E$. It is easily seen that, $\forall \tau, K_{\tau}$ is a compact operator and that the application $\tau \rightarrow K_{\tau}$ is continuous. In the next two lemmae, we derive the a-priori estimates which will enable us to use a topological degree argument: 
Lemma 1.7 : Suppose $u_{a}, v_{a}, \mu_{a}$ is a solution of (1.15). Then:

$\mathrm{v}_{\mathrm{a}} \geq 0$ on $[0, \mathrm{a}], \quad \mathrm{u}^{\prime}{ }_{\mathrm{a}}>0$ and $\mathrm{v}^{\prime}{ }_{\mathrm{a}}<0$ on $[0, \mathrm{a}]$,

$u_{a}$ and $v_{a}$ satisfy the relations. (1.7), (1.8) and (1.9) on $[0, a]$.

Proof : Let us argue by contradiction and suppose that min $v_{a}(x)<0$.
$[0, a]$

Then either there exists $x \in[0, a]$ with $v_{a}(x)<0$ and $v^{\prime}{ }_{a}(x)=0$, or $\forall x \in[0, a]$ such that $v_{a}(x)<0$, one has $v^{\prime}{ }_{a}(x)>0$. In the first case, the maximum principle leads to a contradiction. In the second case, we would have $-\lambda v_{a}^{\prime}(0)+\underset{a}{c}(0)<0$, which is impossible since $-\lambda v^{\prime}{ }_{a}(0)+c v_{a}(0)=c\left(1-z_{0}\right)+\mu_{a}$. Now, since $v_{a} \geq 0$, one has $v^{\prime}{ }_{a}(a)<0$ (thus $v^{\prime}{ }_{a}<0$ on $\left.[0, a]\right)$. Hence, from $-u^{\prime}{ }_{a}+c u_{a}-\lambda v^{\prime}{ }_{a}+c \cdot v=c$ on $[0, a]$, we can deduce $u^{\prime \prime}{ }_{a}(a)>0$ (thus $u^{\prime}{ }_{a}>0$ on $[0, a]$ ). Lastly, according to lemma 1.2 and Corollary $1.3, u_{a}, v_{a}$ satisfy the relations $(1.7)-(1.9)$ on $[0, a]$.

Lema 1.8: $\forall a_{0}>0$, there exists a constant $k\left(=k\left(a_{0}\right)\right)$ such that, $\forall a \geq a_{0}, \forall \tau \in[0,1]$; one has $\left\|u_{a}\right\| \mathscr{C}^{1}([0, a], \mathbb{R}) \leq k$ and $\left\|\cdot v_{a}\right\|_{C^{1}}([0, a], \mathbb{R}) \leq k$, where $\left(u_{a}, v_{a}, \mu_{a}\right)$ is any solution of $(1.15)$.

Proof : Let $a \geq a_{0}>0$. From (1.12),we have:

$\forall x \in[0, a] \quad u_{a}^{\prime 2}(x)=u_{a}^{\prime 2}(0)+2 c \int_{0}^{x} u_{a}^{\prime 2} d s-2 \int_{0}^{x} \tau f\left(u_{a}\right) v_{a} u^{\prime} d s$

We can now deduce,using the lemma 1.7 (and (1.9)), that :

$\forall x \in[0, a] \quad u_{a}^{\prime 2}(x) \geq u_{a}^{\prime 2}(0)-2 \Lambda^{\prime} \tau \int^{1} f(s)(1-s) d s$

Thus, if $u_{a}^{\prime}(0)>{\sqrt{2 \Lambda^{\prime} \tau} \int_{z_{0}}^{l} f(s)(1-s) d s}^{z} c^{z}$, we obtain

$1-z_{0}=u_{a}(a)-u_{a}(0) \geq a \sqrt[0]{u_{a}^{\prime 2}(0)-c_{1}^{2}} \geq a_{0} \sqrt{u_{a}^{\prime 2}(0)-c_{1}^{2}}$ 
that is $: u_{a}^{\prime}(0) \leq \sqrt{c_{1}^{2}+\left(\frac{1-z_{0}}{a_{0}}\right)^{2}}$

Therefore, in any case, we have $u_{a}^{\prime}(0) \leq \sqrt{c_{1}^{2}+\left(\frac{1-z_{0}}{a_{0}}\right)^{2}}$

hence : $u_{a}^{\prime}(0) \leq \sqrt{2 \Lambda^{\prime} \int_{z_{0}}^{l} f(s)(1-s) d s+\left(\frac{1-z_{0}}{a_{0}}\right)^{2}}=\alpha\left(a_{0}\right)$

Notice that $\alpha\left(a_{0}\right)$. does not depend on $\tau$. Besides, from Lemma 1.7 , we know that $z_{0} \leq u_{a} \leq 1, \quad 0 \leq v_{a} \leq \Lambda^{\prime}\left(1-z_{0}\right)$ and $u_{a}^{\prime}>0$ on $[0, a]$. Hence, since $-u_{a}^{\prime}+c u_{a}$ is an increasing function, we obtain $\forall x \in[0, a] u_{a}^{\prime}(x) \leq \alpha\left(a_{0}\right)+c\left(1-z_{0}\right)$. It is now easy to conclude the proof;as $v_{a}^{\prime}=\frac{1}{\lambda}\left(-u_{a}^{\prime}+c u_{a}+c v_{a}-c\right)$ on $[0, a]$.

We can now state an existence result for the problem (1.14) :

Lemma 1.9: $\forall a>0$, there exists a solution $\left(\mathrm{u}_{a}, \mathrm{v}_{a}, \mu_{a}\right)$ of the problem (1.14). Moreover, $\forall \mathrm{a}_{\mathrm{o}}>0, \mathrm{u}_{\mathrm{a}}$ and $\mathrm{v}_{\mathrm{a}}$ are bounded independently of $\mathrm{a} \geq \mathrm{a}_{\mathrm{o}}$ in $\varphi_{,}^{2}([0, \mathrm{a}], \mathbb{R})$.

Proof : Let $a \geq a_{0}>0$. According to the lemmael.7 and 1.8 , we have : $\forall \tau \in[0,1]$, $(0,0,0) \notin\left(\mathrm{I}-\mathrm{K}_{\tau}\right)(\partial \Omega)$, where

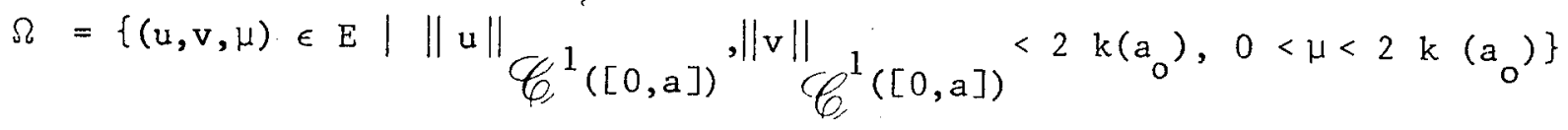

Thus the Leray-Schauder degree of $\left(\mathrm{I}-\mathrm{K}_{\tau}\right)$ with respect to $\Omega$ and 0 is wel1-defined and further is independent of $\tau \in[0,1]$ (see [8] for the definition and the main properties of the Leray-Schauder degree).

It remains to compute this degree (and to show that it is different from 0 ). In order to do this, let us explicitly write down $\Phi_{0}=\mathrm{I}-\mathrm{K}_{0}$ : $\Phi_{\hat{o}}\left((\mathrm{u}, \mathrm{v}, \mu)=\left(\mathrm{u}-\mathrm{U}_{\mathrm{o}}(\mu), \mathrm{v}-\mathrm{V}_{\mathrm{o}}(\mu), \mathrm{u}(\mathrm{a})-1\right)\right.$

where $U_{o}(\mu)$ is the function $: x \rightarrow \frac{\mu}{c}\left(e^{c x}-1\right)$ and $V_{o}(\mu)$ the function $: x \rightarrow\left(1-z_{0}+\frac{\mu}{c}\right)$ $\left(1-e^{\frac{c}{\lambda}(x-a)}\right)$. $\forall t \in[0,1]$, let us introduce the operator $\Phi_{t}: E-E:$

$\Phi_{t}((u, v, \mu))=\left(u-u_{0}(\mu), \dot{v}-v_{0}(\mu),(1-t)(u(a)-1)+t\left(\frac{\mu}{c}\left(e^{c a}-1\right)+z_{0}\right)\right)$

It is easily seen that the map $: t \rightarrow \Phi_{t}$ is continuous and that, $\forall t \in[0,1],(0,0,0) \notin \Phi_{t}$ $(\partial \Omega)$. Therefore $\mathrm{d}\left(\Phi_{0}, \Omega, 0\right)=\mathrm{d}\left(\Phi_{1}, \Omega, 0\right)=1$, since the function $: \mu \rightarrow \frac{\mu}{\mathrm{c}}\left(\mathrm{e}^{\mathrm{ca}}-1\right)+z_{0}$ is increasing. 
Thus we proved $d\left(I-K_{1}, s i, 0\right) \neq 0$. It follows that there exists a solution $\left(u_{a l}, v_{a}, \mu_{a}\right)$ of the problem $(1.14)$ and $u_{a}$ and $v_{a}$ are bounded independently of $a \geq a_{o}$ in $C_{i}^{2}([0, \varepsilon], I R)$.

End of the proof of Proposition 1.6 : We claim that $\left(u_{a}, v_{a}\right)$ defined $:=$ Lemma 1.9 converges as $a \rightarrow+\infty$ to $(u, v)$ satisfying (1.13). Indeed let us extend $u_{a}$ and $v_{a}$ to $\mathbb{R}^{+}$by set.ting $\forall x \geq a u_{a}(x)=1$ and $v_{a}(x)=0$. Since, by Lemma $1.9, u_{a}$ and $v_{a}$ are bounded independent $1 \mathrm{y}$ of $\mathrm{a} \geq \mathrm{a}_{\mathrm{o}}$ in $\mathrm{H}_{1 \mathrm{loc}}^{2}\left(\mathbb{R}^{+}\right)$, there exists a sequence ( $\mathrm{a}_{\mathrm{n}}$ ) with 1 im $\mathrm{a}_{\mathrm{n}}=+\infty$ such that $u_{\mathrm{a}} \rightarrow \mathrm{u}$ and $\mathrm{v}_{\mathrm{a}} \rightarrow \mathrm{v}$ in $\mathscr{C}_{10 \mathrm{c}}^{1}\left(\mathbb{R}^{+}\right)$.

We remark that $(u, v)$ satisfy;

and

$$
\left\{\begin{array}{l}
-u^{\prime \prime}+c u^{\prime}=f(u) v, \quad \text { on } \mathbb{R}^{+} \\
-\lambda v^{\prime \prime}+c v^{\prime}=-f(u) v
\end{array}\right.
$$

$$
\left\{\begin{array}{l}
-u^{\prime}+c u-\lambda v^{\prime}+c v=c \text { on } \mathbb{R}^{+} \\
u^{\prime} \geq 0 \text { and } z_{0} \leq u<1, v^{\prime} \leq 0 \text { and } 0 \leq v \leq \Lambda^{\prime}\left(1-z_{o}\right) \\
u(0)=z_{0}, \quad v(0) \geq \Lambda\left(1-z_{0}\right)
\end{array}\right.
$$

We can now see that $\ell_{1}=\mathrm{u}(+\infty)=1$ and $\ell_{2}=\mathrm{v}(+\infty)=0$. Indeed, integrating the equation satisfied by $u(\operatorname{resp} v)$, we get that $u^{\prime}(+\infty)=1$ im $u^{\prime}(x)\left(\right.$ resp $v^{\prime}(+\infty)$ ) exists and hence $x \rightarrow+\infty$

is equal to 0 . Then, the equation satisfied by $u$ yields $u^{\prime \prime}(+\infty)=0$ and $f\left(l_{1}\right) l_{2}=0$.

Therefore, as $z_{0} \leq \ell_{1} \leq 1$, we obtain $\ell_{2}=0$, and thus $\ell_{1}=1$, since $-u^{\prime}(+\infty)+c u(+\infty)-$ $\lambda v^{\prime}(+\infty)+c v(+\infty)=c$.

$(u, v)$ can be extended in a unique way to a maximal solution, denoted again by $(u, v)$, of :

$$
\left\{\begin{array}{l}
-u^{\prime \prime}+c u^{\prime}=f(u) v \\
-\lambda v^{\prime \prime}+c v^{\prime}=-f(u) v
\end{array}\right.
$$

We know that $v \geq 0$ on $\mathbb{R}^{+}$and $v(0)>0$. On the other hand, according to Lemma 1.2 , if $v \geq 0$ on $\left[x_{0},+\infty\left[\right.\right.$ for some $x_{0} \in \mathbb{R}$, then $v^{\prime} \leq 0$ on $\left[x_{0},+\infty[\right.$. Therefore obviously we have $v(x) \geq 0$. $\forall x$. Then it is easily seen that $u$ and $v$ are defined on $\mathbb{R}$ and that Lemma 1.2 and Corollary 1.3 can be applied to $(u, v)$ on $\mathbb{R}$.

Finally it can be proved easily that $u^{\prime}>0$ and $v^{\prime}<0$ on $\mathbb{R}$. 
Notice that the couple $(u, v)$ defined by Proposition 1.6 is solution of $(0.1)$ if and only if $u(-\infty)=0$ and $v(-\infty)=1$. In the next two remarks we derive very simple conditions for a couple $(u, v)$ satisfying (1.13) to be a solution of (0.1).

Remark 1.10 : Let $(u, v)$ satisfy $(1,13)$. There are two prossibilities : either there exists $\underline{x}<0$ with $u(\underline{x})=0$, or $\forall x<0$ one has $u(x)>0$. In the latter case it is easily shown that $(u, v)$ is a solution of $(0.1)$; in this situation, we set $\underline{x}=-\infty$. Thus, in any case,there exists an unique $\underline{x} \in[-\infty, 0[$ such that $u(\underline{x})=0$; then one has $\forall x \in] \underline{x},+\infty\left[u^{\prime}(x)>0\right.$ and $u \in \ell^{0}([\underline{x},+\infty],[0,1])$. Moreover $u^{\prime}(\underline{x})=0 \Leftrightarrow \underline{x}=-\infty \Leftrightarrow$ $(u, v)$ is solution of $(0.1)$.

Remark 1.11 :To $(\mathrm{u}, \mathrm{v})$ satisfying (1.13) we shall henceforth associate the unique function $\mathrm{h} \in \mathscr{C}([0,1]) \cap \mathscr{C}_{1}^{1}(] 0,1[)$ defined by :

$$
\begin{cases}\forall s \in] 0,1[ & h(s)=u^{\prime}\left(u^{-1}(s)\right) \text { and } \frac{d}{d s} h(s)=c-\frac{v\left(u^{-1}(s)\right) f(s)}{h(s)}, \\ h(1)=0, & h(0)=u^{\prime}(\underline{x}),\end{cases}
$$

where $\underline{x}$ is defined in Remark 1.10. Then $h(0)=0 \Leftrightarrow(u, v)$ is a solution of $(0.1)$.

We are now ready to complete the proof of Theorem 1.1 (ii). Let $\left.z_{0} \in\right] 0,1[$ be fixed. For any $c>0$ let us consider the couple $(u, v)$ defined by Proposition 1.6 and the function $h$ associated to $(u, v)$ by $(1.16)$. We have :

$\forall s \in] 0,1\left[\cdot h^{\prime}(s) \geq c-\frac{\Lambda^{\prime} f(s)(1-s)}{h(s)} \geq c-\frac{\Lambda^{\prime} \sigma s}{h(s)}\right.$

We claim that, if $c \geq 2 \sqrt{\sigma \Lambda^{\prime}}$, one has $h(0)=0$ (this will complete the proof according to Remark 1.11). Indeed let $c \geq 2 \sqrt{\sigma \Lambda^{\prime}}$. The linear function $H(s)=\frac{c+\sqrt{c^{2}-4 \Lambda^{\prime} \sigma}}{2} s$ is such that $\left.\forall s \in\right] 0,1\left[\quad H^{\prime}(s)=c-\frac{\Lambda^{\prime} \sigma_{s}}{H(s)}\right.$. Thus, since $h(1)<H(1)$, it is easily shown that $h \leq H$ on $] 0,1]$. Hence $h(0) \leq H(0)=0$.

c) Proof of Theorem 1.1 (iii)

One has the following more general result :

Lemma 1.12: We assume that the condition (0.4) halds. We suppose furthormore that $f$ is of class $C^{1}$ in a neighbourhood of 1 . Then $\left.\forall z \in\right] 0,1[$, $\forall c>0$, there exists one and only one couple $(u, v)$ satisfying $(1.13)$. 
Remarik 1.13: Proposition 1.6 dealt with the existence of such a couple (u,v). We shall here check the uniqueness under an additional hypothesis of regularity on $f$ in a neighbourhood of 1 .

Proof : To prove Lemma 1.12, we replace the system of two differential equations of the second order considered up to now by a system of three differential equations of the first order. Then we perform a local analysis about a singular point, which will enable us to prove uniqueness.

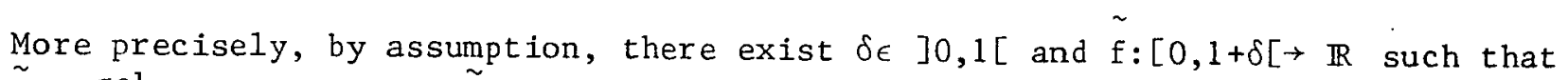
$\tilde{f} \in \mathscr{C}, 1](1-\delta, 1+\delta[)$ and $\tilde{f}=f$ on $[0,1]$. Let us introduce the vector field $F$ defined on $] 0,1+\delta[\times \mathbb{R} \times \mathbb{R}$ by :

$$
F\left(\left(x_{1}, x_{2}, x_{3}\right)\right)=\left(c\left(x_{1}-1\right)+x_{3}, \frac{c}{\lambda} x_{2}-\frac{1}{\lambda} x_{3},-\tilde{f}\left(x_{1}\right) x_{2}\right)
$$

Let $(u, v)$ be a couple satisfying (1.13). Let us denote $w \equiv-\lambda v^{\prime}+c v$. One has $\forall x \in \mathbb{R} \quad w^{\prime}(x)<0$, and $w(+\infty)=0$. Thus $X=(u, v, w)$ is such that :

$$
\left\{\begin{array}{l}
\frac{d X}{d x}=F(X) \text { on } \mathbb{R}, \\
X(+\infty)=(1,0,0), \\
\exists x_{0}, \quad \forall x \geq x_{0}, \quad X(x) \in\left\{\left(x_{1}, x_{2}, x_{3}\right) \mid x_{1}<1, x_{2}>0, x_{3}>0\right\} .
\end{array}\right.
$$

Next consider the singular point $(1,0,0)$; the derivative DF $((1,0,0))$ of $F$ at $(1,0,0)$ has two positive eigenvalues and one negative eigenvalue. Thus the stable Manifold Theorem (see [37) yields the uniqueness (up to a translation of the origin) of a vector field $x \in C^{1}\left(\mathbb{R}, \mathbb{R}^{3}\right)$ for which (1.18) holds.

This implies that, if $\left(u_{i}, v_{i}\right), i=1,2$, satisfy (1.13), then there exists $\delta \in \mathbb{R}$ such that $\forall x \in \mathbb{R} \quad u_{1}(x)=u_{2}(x+\delta)$. and $v_{1}(x)=v_{2}(x+\delta)$. It is now straightfcrward to conclude the proof, since, $\forall i=1,2, u_{i}^{\prime}>0$ and $u_{i}(0)=z_{0}$.

The proof of Theorem 1.1 is now complete.

\subsection{The role of the ignition temperature.}

In this section, we show that we can obtain a solution of (0.1) by passing to the limit as $v \rightarrow U$ in the solutions of the model with ignition temperature. We then 
study the asymptotic behaviour of this sclution, as the activation enerzy goes to $+\infty$.

\subsubsection{Construction of a solution of $(0.1)$.} blem :

Let us fix some $\left.z_{0} \epsilon\right] 0,1[$ and, for any $\theta \in] 0,1[$, let us consider the pro-

$$
(1.19)\left\{\begin{array}{c}
\text { To find } u, v \in C^{1}(\mathbb{R},[0,1]) \text { and } c \geq 0 \text { such that : } \\
-u^{\prime \prime}+c u^{\prime}=f(u) x[\theta, 1](u) v, \\
-\lambda v^{\prime \prime}+c v^{\prime}=-f(u) x_{[\theta, 1]}(u) v, \\
u(-\infty)=0, \quad u(0)=z_{0}, u(+\infty)=1, \\
v(-\infty)=1, \quad v(+\infty)=0,
\end{array}\right.
$$

where $x_{[\theta, 1]}$ denotes the characteristic function of $[\theta, 1]$ :

From the study of the model with ignition temperature (see [1]), we know that, $\forall \theta \in] 0,1\left[,(1.19)\right.$ has got a solution $\left(u_{\theta}, v_{\theta}, c_{\theta}\right)$; moreover :

$$
\left\{\begin{array}{l}
0<u_{\theta}, v_{\theta}<1,0<u_{\theta}^{\prime} \leq c_{\theta},-\frac{c_{\theta}}{\lambda} \leq v_{\theta}^{\prime}<0 \text { on } \mathbb{R}, \\
\left.c^{2} \geq 2 \Lambda \int_{\theta}^{1} f(s)(1-s) d s \quad \text { (where } \Lambda \text { is given by }(1.2)\right), \\
u_{\theta} \text { and } v_{\theta} \text { satisfy the relations }(1.7),(1.8) \text { and (1.9). }
\end{array}\right.
$$

Our main result is then the following :

Theorem 1.14: Under condition (0.4), $\left.\forall z_{0} \in\right] 0,1[$, there exists a sequence $\left(\theta_{n}\right)_{n}$, with $\lim \theta_{n}=0$, such that the solution $\left(u_{\theta_{n}}, v_{\theta_{n}}, c_{\theta_{n}}\right)$ of (1.19) converges to a solution $\left(u_{0}, v_{0}, c_{0}\right)$ of $(0.1)$ in $\varphi_{1}^{2}(\mathbb{R}) \times \varphi^{2}(\mathbb{R})^{n} \times \mathbb{R}$.

Proof : According to the results that we recalled above, it suffices to prove that $c_{\theta}$ is bounded independently of $\left.\theta \epsilon\right] 0,1\left[\right.$, in order to obtain estimates of $\left(u_{\theta}, v_{\theta}, c_{\theta}\right)$ independent of $\theta \leq \theta_{0}$. This will be proved in the next lemma : 
Lemma 1.15 $: \forall \theta \in] 0,1\left[\quad c_{\theta}<2 \sqrt{\Lambda_{x \in] 0,1[}^{\prime} \operatorname{sup.}_{x \in 0} \frac{f(x)(1-x)}{x}}\right.$.

Proof $: \forall \theta \in] 0,1\left[\right.$, let us associate to $\left(u_{\theta}, v_{\theta}, c_{\theta}\right)$ the unique function $h_{\theta} \in C^{0}$ $([1,1]) \cap \mathscr{C}^{1}([0,1[-\{\theta\})$ defined by :

$$
(1.20) \begin{cases}\forall s \in] 0,1\left[\quad h_{\theta}(s)=u_{\theta}^{\prime}\left(u_{\theta}^{-1}(s)\right), \quad h_{\theta}(1)=h_{\theta}(0)=0,\right. \\ \forall s \in[\theta, 1] \quad, h_{\theta}^{\prime}(s)=c_{\theta}-\frac{f(s) v_{\theta}\left(u_{\theta}^{-1}(s)\right)}{h_{\theta}(s)} .\end{cases}
$$

Since $h_{\theta}(\theta)=c_{\theta} \theta$ and $h_{\theta}(1)=0$, there exists $\left.\theta_{1} \in\right] 0,1\left[\right.$ such that $\forall s \in\left[\theta, \theta_{1}\left[h_{\theta}(s)>\frac{c_{\theta}}{2} s\right.\right.$ and $h_{\theta}\left(\theta_{1}\right)=\frac{c_{\theta}}{2} \theta_{1}$. We remark that $h^{\prime}\left(\theta_{1}\right) \leq \frac{c_{\theta}}{2}$. On the other hand we know, from the equation satisfied by $h_{\theta}$, that $: h_{\theta}^{\prime}\left(\theta_{1}\right) \geq c_{\theta}-\frac{\Lambda^{\prime} f\left(\theta_{1} j\left(1-\theta_{1}\right)\right.}{h_{\theta}\left(\theta_{1}\right)}$. Combining these
two inequalities, we conclude the proof.

Lemma 1.15 implies the existence of a sequence $\left(\theta_{n}\right)$ with $\lim \theta_{n}=0$ such that $\left(u_{\theta_{n}}, v_{\theta_{n}}, c_{\theta_{n}}\right)$ converges to $\left(u_{o}, v_{o}, c_{o}\right)$ in $\mathscr{C}_{10 c}^{l}(\mathbb{R}) \times \varphi_{1 o c}^{l}(\mathbb{R}) \times \mathbb{R}$.

It remains to show that ( $\left.u_{0}, v_{0}, c_{0}\right)$ is a solution of $(0.1)$.

$\forall \mathrm{n}$, let us define $\mathrm{x}_{\mathrm{n}}$ by $u_{\theta_{\mathrm{n}}}\left(\mathrm{x}_{\mathrm{n}}\right)=\theta_{\mathrm{n}}$. We claim that lim $\mathrm{x}_{\mathrm{n}}=-\infty$. Indeed, should there exist a subsequence $\left(\mathrm{x}_{\mathrm{n}_{\mathrm{k}}}\right)$ with $\lim \mathrm{x}_{\mathrm{n}_{\mathrm{k}}}=\tilde{\mathrm{x}}>-\infty$, then we would have necessarily:

$$
\left\{\begin{array}{l}
-u_{0}^{\prime \prime}+c_{0} u_{0}^{\prime}=f\left(u_{0}\right) v_{0} \text { on } \mathbb{R} \\
\left.\left.u_{0}(0)=z_{0}, \quad u_{0}=0 \text { sur }\right]-\infty, \tilde{x}\right]
\end{array}\right.
$$

which is impossible, by the uniqueness in the initial value problem for O.D.E.. Now, since $\lim x_{n}=-\infty$, we see that :

$$
\left\{\begin{array}{l}
-u_{0}^{\prime \prime}+c_{0} u_{o}^{\prime}=f\left(u_{0}\right) v_{0} \\
-\lambda v_{0}^{\prime \prime}+c v_{0}^{\prime}=-f\left(u_{o}\right) v_{0}
\end{array} \text { on } \mathbb{R}\right.
$$


On the other hand, from the qualitative properties satisfied by $\left(u_{\theta}, v_{\theta}, c_{\theta}\right)$, we deduce :

$$
\left\{\begin{array}{l}
-u_{0}^{\prime}+c_{0} u_{0}-\lambda v_{0}^{\prime}+c_{0} v_{0}=c_{0} \text { on } \mathbb{R}, \\
0 \leq u_{0} \leq 1, \quad u_{0}^{\prime} \geq 0, \quad 0 \leq v_{0} \leq 1, \quad v_{0}^{\prime} \leq 0 \text { on } \mathbb{R}, \\
u_{0}(0)=z_{0}
\end{array}\right.
$$

This implies, as above, (see proposition 1.6), that $\left(u_{0}, v_{0}, c_{0}\right)$ is a solution of $(0.1)$. Finally, it is straightforward to prove the convergence in $\oint^{2}(\mathbb{R}) \times C^{2}(\mathbb{R}) \times \mathbb{R}$

Theorem 1.14 asserts that by truncating $f$ by 0 on $[0, \theta]$ and then by letting $\theta \rightarrow 0$ in the model with ignition temperature, one can construct a solution of (0.1); in other words, there exists a solution $\left(u_{0}, v_{0}, c_{0}\right)$ of $(0.1)$ haying the following property (denoted by $(P))$ :

There exists a sequence $\left(\theta_{n}\right)_{n}$ with 1 im $\theta_{n}=0$ such that the solution $\left(u_{\theta_{n}}, v_{\theta_{n}}, c_{\theta_{n}}\right)$ of $(1.19)$ converges to $\left(u_{o}, v_{o}, c_{o}\right)$ in $\varphi_{,}^{2}(\mathbb{R}) \times \varphi_{,}^{2}(\mathbb{R}) x \mathbb{R}$.

Actually, one does not know whether there exists or not a unique solution of (0.1) with the property (P). We will see in Section 2.2 .2 below, that, at least in the case $\lambda<1$, there is one and only one solution of (0.1) satisfying (P).

Anyway, in the remainder of this section, we will show a particular property of the solutions of $(0.1)$ satisfying $(P)$, which will prove to be of crucial interest in the asymptotic analysis (see Section 1.2 .2 below).

Proposition 1.16: Let condition (0.4)hold. We assume furthermore that $\mathrm{f}$ is of class $C_{1}{ }^{1}$ in a neighbourhood of 1 and that $\mathrm{f}$ is differentiable at 0 with $\mathrm{f}^{\prime}(0)=0$. Let $\left(\mathrm{u}_{0}, \mathrm{v}_{0}, \mathrm{c}_{0}\right)$ be a solution of $(0.1)$ satisfying the property $(\mathrm{P})$. Then the function $h_{0}$ associated to $\left(u_{0}, v_{0}, c_{0}\right)$ by $(1.16)$ is differentiable at 0 and $h_{0}^{\prime}(0)=c_{0}$.

Remark 1.17 : This proposition generalizes a previously known result for the scalar case $(\lambda=1)$ (see, for example, Johnson [5], Marion[7], Uchiyama[9]). 
Proof of Proposition 1.16: Let $\left(u_{0}, v_{0}, c_{0}\right)$ satisfy $(P) ;$ let us denote $c_{n}=c_{\theta_{n}}$. By considering the equation satisfied by $h_{0}$, it is easily seen that $h_{0}^{\prime}(0)$ exists and is equal either to $c_{0}$ or to 0 .

Thus it remains to show that $h_{0}^{\prime}(0)=c_{0}$. We first remark that (P) implies that $\forall s \in] 0,1\left[h_{0}(s)=1\right.$ im $h_{\theta_{n}}(s)$ (where $h_{\theta}$ is defined by (1.20)).

Next, let us associate to $c_{n}$ the unique couple $\left(u_{n}, v_{n}\right)$ satisfying (1.13) (see Lemma 1.12) and let us introduce also the function $h_{n}$ associated to $\left(u_{n}, v_{n}, c_{n}\right)$ by $(1.16)$. Let $x_{n}$ be such that $u_{\theta_{n}}\left(x_{n}\right)=\theta_{n}$. Then, according to the Stable Manifold Theorem, we must have $u_{n}=u_{\theta_{n}}$ on $\left[x_{n}+\infty\right]$. Thus $\tilde{h}_{n}=h_{\theta_{n}}$ on $\left[\theta_{n}, l\right]$.

These two remarks imply, on one hand, that $\tilde{h}_{n}\left(\theta_{n}\right)=c_{n} \theta_{n}$, and, on the other hand, that $\left.\forall_{s \in}\right] 0,1\left[h_{0}(s)=\lim \tilde{h}_{n}(s)\right.$. We can now easily show that $h_{0}^{\prime}(0)=c_{0}$. Indeed, since $\tilde{h}_{n}\left(\theta_{n}\right)=c_{n} \theta_{n}$ and $\tilde{h}_{n}^{\prime}<c_{n}$ on $] 0,1\left[\right.$, one has $\tilde{h}_{n}(0)>0$. As $\tilde{h}_{n}(1)=0$, there exists $y_{n}$ with the property that $\tilde{h}_{n}\left(y_{n}\right)=\frac{c_{n}}{2} y_{n}$ and $\forall s \in\left[0, y_{n}\left[\tilde{h}_{n}(s)>\frac{c_{n}}{2} s\right.\right.$. We claim that there are $\alpha>0$ and $n_{0} \in \mathbb{N}$ such that, $\forall n \geq n_{0}$, one has $y_{n} \geq \alpha$. Indeed, arguing by contradiction, there exists a subsequence of $\left(\dot{y}_{n}\right)_{n}$, again denoted $\left(y_{n}\right)_{n}$, with $\lim y_{n}=0$. Since, $\forall n, \quad \tilde{h}_{n}^{\prime}\left(y_{n}\right) \geq c_{n}-\frac{f\left(y_{n}\right)\left(1-y_{n}\right)}{\tilde{h}_{n}\left(y_{n}\right)}$, this yields 1 im $\tilde{h}_{n}^{\prime}\left(y_{n}\right) \geq c_{0}$, which is impossible, since, $\forall n, \tilde{h}_{n}^{\prime}\left(y_{n}\right) \leq \frac{c}{2}$. Now, $\left.\forall s \in\right] 0, \alpha\left[, h_{0}(s)=\lim \tilde{h}_{n}(s) \geq \frac{c_{0}}{2} s\right.$, and thus $h_{0}^{\prime}(0)=c_{0}$. The proof of Proposition 1.16 is thereby complete.

\subsubsection{Asymptotic analysis}

In this section, we assume that $f$ depends on a parameler $\varepsilon>0$ and we let $\mathrm{f}=\mathrm{f}_{\varepsilon}$. The corresponding solution of $(0.1)$ obtained in theorem 1.14 (for some fixed $\lambda>0$ ) will be denoted by $\left(u_{o, \varepsilon}, v_{o, \varepsilon}, c_{o, \varepsilon}\right)$ and we shall investigate the asymptotic behaviour of this solution as $\varepsilon \rightarrow 0$. The following conditions will be assumed on the family of functions $f_{\varepsilon}$ :

(1.21) For each $\varepsilon>0, f_{\varepsilon}$ satisfies condition $(0.4)$,

'(1.22) For each $\varepsilon>0, f_{\varepsilon}$ is of class $\mathscr{C}^{l}$ in a neighbourhood of 1 and $\mathrm{f}_{\varepsilon}$ is differentiable at 0 with $f_{\varepsilon}^{\prime}(0)=0$, 
(1.23)

$$
{ }^{\exists \theta_{\varepsilon}}, \quad 0 \leq \theta_{\varepsilon} \leq i \text { such that } \lim _{\varepsilon \downarrow 0} \dagger_{\varepsilon} \theta_{\varepsilon}=1 \text { and }
$$

$$
\lim _{\varepsilon \rightarrow 0}\left\{\sup _{\left.x \in] 0, \theta_{\varepsilon}\right]} \frac{f_{\varepsilon}(x)(1-x)}{x}\right\}=0,
$$

(1.24) $\lim _{\varepsilon \rightarrow 0} \int_{0}^{1} f_{\varepsilon}(s)(1-s) d s=m \cdot 0, m<+\infty$.

Remark 1.18 : The typical function $\mathrm{f}_{\varepsilon}$, provided by the Arrhenius law, is $\mathrm{f}_{\varepsilon}(\mathrm{x})=\frac{1}{\varepsilon^{2}} \exp \left(\frac{1}{\varepsilon} \frac{\mathrm{x}-1}{\mathrm{x}}\right)$. Recall that $\varepsilon$ represents the inverse of the activation energy.

Our main result is the following :

Theorem 1.19: Under conditions (1.21), (1.22), (1.23), (1.24) and with the above notations :

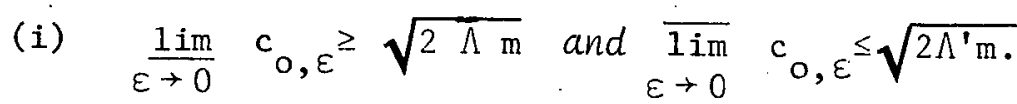

(ii) Let $\left(\varepsilon_{n}\right)_{n}$ be a sequence with the property that lim $\varepsilon_{n}=0$ and $c_{0, \varepsilon}$ converges to some $c_{0}$. Then $\left(u_{0, \varepsilon_{n}}, v_{o, \varepsilon_{n}}\right)$ converges to $\left(u_{o}, v_{0}\right)$ in $\mathscr{C}^{0}(\mathbb{R}) \times \mathscr{C}^{0}(\mathbb{R})$ where $\left(u_{0}, v_{0}\right)$ is uniquely determined by:

$$
\left\{\begin{array}{l}
-u_{0}^{\prime \prime}+c_{0} u_{0}^{\prime}=c_{0} \delta_{x}=x_{0}, \\
-\lambda v_{0}^{\prime \prime}+c_{0} v_{0}^{\prime}=-c_{0} \delta_{x}=x_{0} \\
u_{0}(-\infty)=0, \quad u_{0}(+\infty)=1, \\
v_{0}(-\infty)=1, \quad v_{0}(+\infty)=0,
\end{array}\right.
$$

and $\mathrm{x}_{\mathrm{o}}$ is uniquely determined by the condition $\mathrm{u}_{0}(0)=\mathrm{z}_{0}$.

Remark 1.20: A considerable part of the literature on combustion is devoted to formal asymptotic limits for infinite activation energy (see, for example, Buckmaster and Ludford [2] or Williams [10]). Theorem 1.19 proves that the solution 
( $\left.u_{o, \varepsilon}, \mathrm{v}_{o, \varepsilon}, \mathrm{c}_{o, \varepsilon}\right)$ has got the expected asymptotic behaviour. This result is analogous to the one obtained by Berestycki, Nicolaenko and Scheurer [1] for the model with ignition temperature.

The asymptotic analysis of the other solutions of $(0.1)$, is an open question. A partial answer will be given, in Section 2.3 below, in the case $\lambda<1$.

Remark 1.21 : Based on the formal asymptotic calculations, we conjecture that $c_{0}=\sqrt{\frac{2 m}{\lambda}}$.

Remark 1.22 : The assumptions of Theorem 1.19 could probably be weakened.

Proof of Theorem 1.19: First, recall that, from Lemma 1.5, we know that

$\forall \varepsilon>0 \quad c_{o, \varepsilon}>\sqrt{2 \Lambda \int_{0}^{l} f_{\varepsilon}(s)(1-s) d s}$, which implies :

$\lim _{\varepsilon \rightarrow 0} c_{o, \varepsilon} \geq \sqrt{2 \Lambda \mathrm{m}}$

$\forall \varepsilon>0$, let us denote by $h_{o, \varepsilon}$ the function associated to $\left(u_{0, \varepsilon}, v_{o, \varepsilon}, c_{o, \varepsilon}\right)$ by $(1.16)$. In the next lemma, we investigate the asymptotic limit of $h_{0, \varepsilon}$, as $\varepsilon \rightarrow 0$, and we prove the second part of (i).

Lemma 1.23 : $\forall s \in\left[0,1\left[\lim _{\varepsilon \rightarrow 0}\left(h_{o, \varepsilon}(s)-c_{o, \varepsilon} s\right)=0\right.\right.$,

$$
\varlimsup_{\varepsilon \rightarrow 0} c_{o, \varepsilon} \leq \sqrt{2 \Lambda^{\prime} m}
$$

Proof $: \forall \varepsilon>0$, let us set $\sigma(\varepsilon)=\sup _{\left.x \in] 0, \theta_{\varepsilon}\right]} \frac{f_{\varepsilon}(x)(1-x)}{x} \cdot h_{0, \varepsilon}$ satisfies :

$\left.\forall s \in] 0, \theta_{\varepsilon}\right] h_{o, \varepsilon}^{\prime}(s) \geq c_{o, \varepsilon}-\frac{\Lambda^{\prime} f_{\varepsilon}(s)(1-s)}{h_{o, \varepsilon}(s)} \geq c_{o, \varepsilon}-\frac{\Lambda^{\prime} \sigma(\varepsilon) s}{h_{o, \varepsilon}(s)}$

Since, from Proposition 1.16, one has $h_{0, \varepsilon}^{\prime}(0)=c_{0, \varepsilon}$, it is easily seen as above (see the last part of the proof of Theorem 1.1 (ii)) that :

$\forall s \in\left[0, \theta_{\varepsilon}\right] \quad h_{o, \varepsilon}(s) \geq \frac{1}{2}\left(c_{o, \varepsilon}+\sqrt{c_{o, \varepsilon}^{2}-4 \Lambda^{\prime} \sigma(\varepsilon)}\right) s$ 
Therefore, we have :

$\forall s \in\left[0, \theta_{\varepsilon}\right], 0 \geq h_{0, \varepsilon}(s)-c_{0, \varepsilon} s \geq \frac{1}{2}\left(-c_{0, \varepsilon}+\sqrt{c_{o, \varepsilon}^{2}-4 \Lambda^{\prime} \sigma(\varepsilon)}\right) s$

Thus, since we have $\frac{\lim }{\varepsilon \rightarrow 0} c_{o, \varepsilon}>0$ and $\lim _{\varepsilon \rightarrow 0} \sigma(\varepsilon)=0$, we obtain on one hand that $\lim _{\varepsilon \rightarrow 0}$ $\left(h_{o, \varepsilon}\left(\theta_{\varepsilon}\right)-c_{o, \varepsilon} \theta_{\varepsilon}\right)=0$ and on the other hand that $\forall s \in\left[0,1\left[\lim _{\varepsilon \rightarrow 0}\left(h_{0, \varepsilon}(s)-c_{o, \varepsilon} s\right)=0\right.\right.$.

It remains to show that $\overline{\lim }_{\varepsilon \rightarrow 0} \cdot c_{0, \varepsilon} \leq \sqrt{2 \Lambda^{\prime} \mathrm{m}} \cdot \forall \varepsilon>0$ let $\mathrm{x}_{\varepsilon}$ be defined by $\mathrm{u}_{0, \varepsilon}\left(\mathrm{x}_{\varepsilon}\right)=\theta_{\varepsilon}$. Since $u_{o, \varepsilon}^{\prime}\left(x_{\varepsilon}\right)=h_{o, \varepsilon}\left(\theta_{\varepsilon}\right)$, we deduce from above that $\overline{\lim } c_{o, \varepsilon}=\overline{\operatorname{Iim}} u_{o, \varepsilon}^{\prime}\left(x_{\varepsilon}\right)$. Now, writing (1.12) with $a=x_{\varepsilon}$ and $b=+\infty$, we derive : $\frac{1}{2} u_{o, \varepsilon}^{\prime}\left(x_{\varepsilon}\right)^{2} \leq \Lambda^{\prime} \int_{\theta_{\varepsilon}}^{l} f_{\varepsilon}(s)(1-s) d s$ Therefore one has $: \overline{\lim }_{\varepsilon \rightarrow 0} u_{o, \varepsilon}^{\prime}\left(x_{\varepsilon}\right) \leq \sqrt{2 \Lambda^{\prime} m}$. The proof of the lemma is thereby
complete.

We are now ready to complete the proof of Theorem 1.19. Let $\left(\varepsilon_{n}\right)_{n}$ be a sequence with the property that $c_{0, \varepsilon_{n}}$ converges to some $c_{0}$. We shall denote by $\mathrm{u}_{\mathrm{n}}=\mathrm{u}_{0, \varepsilon_{\mathrm{n}}}, \mathrm{v}_{\mathrm{n}}=\mathrm{v}_{\mathrm{o}, \varepsilon_{\mathrm{n}}}, \mathrm{f}_{\mathrm{n}}=\mathrm{f}_{\varepsilon_{\mathrm{n}}}$ and $\mathrm{x}_{\mathrm{n}}=\mathrm{x}_{\varepsilon_{\mathrm{n}}}$ (we recall that $\mathrm{u}_{\mathrm{o}, \varepsilon_{\mathrm{n}}}\left(\mathrm{x}_{\varepsilon_{\mathrm{n}}}\right)=\theta_{\varepsilon_{\mathrm{n}}}$ ). We claim that there exist $n_{0} \in \mathbb{N}$ and $y \in \mathbb{R}$ such that $\forall n \geq n_{0}$ one has $x_{n} \leq y$. Indeed by setting $a=0$ and $b=x$ in (1.12), we obtain: $\forall x \in\left[0, x_{n}\right] \quad \frac{1}{2} u_{n}^{2}(x) \geq$ $\frac{1}{2} u_{n}^{\prime}{ }^{2}(0)-\Lambda^{\prime} \int_{z_{0}}^{\theta} \varepsilon_{n} f_{n}(s)(1-s) d s$. According to Lemma 1.23 , 1im $u_{n}^{\prime}(0)=c_{0} z_{0}$. Since $\lim \int_{z_{0}}^{\theta} \varepsilon_{n} f_{n}(s)(1-s) d s=0$, we see that there exist $n>0$ and $n_{0} \in \mathbb{N}$ such that $\forall n \geq n_{0}, \forall x \in\left[0, x_{n}\right]$, one has $u_{n}^{\prime}(x) \geq n$. This proves our claim, since $\forall n \geq n_{0}$ $1-z_{0} \geq u_{n}\left(x_{n}\right)-u_{n}(0) \geq n x_{n}$

We can now see that $u_{n} \rightarrow 1$ in $C_{0}^{0}\left(\left[y,+\infty[)\right.\right.$. Moreover, from (1.9), we obtain $v_{n} \rightarrow 0$ in $\ell^{0}([y,+\infty[)$.

Next, for any $k \in \mathbb{N}^{*}$, let us consider the interval $[-k, y]$. Then, from Lemma 1.4 , we know that $u_{n}$ and $v_{n}$ are bounded in $H^{1}([-k, y])$ independent of $n \geq n_{1}$. Hence there exists a subsequence denoted by $\left(n_{\ell}\right)_{\ell}$, such that $u_{n_{\ell}}, v_{n_{\ell}}$ converge weakly in 
$H^{1}([-k, y])$ and strongly in $C_{0}^{0}([-k, y])$ to $u_{0}, v_{0}$ and, moreover, $x_{n_{l}} \rightarrow x_{0}$. We remark that $u_{0}=1$ and $v_{0}=0$ on $\left[x_{0}, y\right]$ (hence $x_{0}>0$ since $\left.u_{0}(0)=z_{0}\right)$. Besidés, from (1.23), we know that $u_{n_{\ell}}$ and $v_{n_{\ell}}$ are bounded in $H^{2}\left(\left[-k, x_{0}-\delta\right]\right)$ independent of $\ell \geq l_{1}$. Thus we can derive $-u_{0}^{\prime \prime}+c_{0} u_{0}^{\prime}=0$ and $-\lambda v_{0}^{\prime \prime}+c_{0} v_{0}^{\prime}=0$ on $\left[-k, x_{0}[\right.$. Lastly we know from Lerma 1.23 that $u_{0}^{\prime}(0)=\lim u_{n}^{\prime}(0)=c_{0}{ }_{0}$ and we remark that :

$-u_{0}^{\prime}(0)+c_{0} u_{0}(0)-\lambda v_{0}^{\prime}(0)+c_{0} v_{0}(0)=\lim \left(-u_{n_{\ell}}^{\prime}(0)+c_{n_{\ell}} u_{n_{\ell}}(0)-\lambda v_{n_{\ell}}^{\prime}(0)+c_{n_{\ell}} v_{n_{\ell}}(0)\right)=c_{0}$ Hence, finally, $\left(u_{0}, v_{0}\right)$ satisfies :

$$
\left\{\begin{array}{l}
-u_{0}^{\prime}+c_{0} u_{0}=0 \text { and }-\lambda v_{0}^{\prime}+c_{0} v_{0}=c_{0} \text { on }\left[-k, x_{0}[,\right. \\
u_{0}=1 \text { and } v_{0}=0 \text { on }\left[x_{0}, y\right]
\end{array}\right.
$$

Since $u_{0} \epsilon \epsilon^{0}([-n, y])$, we see that $x_{0}=-\frac{1}{c_{0}} \log z_{0}$ and so $x_{0}$ and $\left(u_{0}, v_{0}\right)$ are independent of the subsequence $\left(n_{\ell}\right)$ considered.

Thus, we have shown that $\forall k \in \mathbb{N}^{*}, u_{n} \rightarrow u_{0}$ and $v_{n} \rightarrow v_{0}$ in $\ell_{(}^{0}\left(\left[-k,+\infty[)\right.\right.$ where $u_{0}, v_{0}$ are uniquely determined by :

$$
\left\{\begin{array}{l}
\left.-u_{0}^{\prime}+c_{0} u_{0}=0 \text { and }-\lambda v_{0}^{\prime}+c_{0} v_{0}=c_{0} \text { on }\right]-\infty, x_{0}[, \\
u_{0}=1 \text { and } v_{0}=0 \text { on }\left[x_{0}+\infty[\right.
\end{array}\right.
$$

and $x_{0}$ is uniquely determined by the condition $u_{0}(0)=z_{0}$.

It is straightforward to show the convergence in $\mathscr{C}^{0}(\mathbb{R})$; this concludes the proof of Theorem 1.19 .

\subsection{The problem on a bounded interva1.}

From the viewpoint of the numerical approximation, it is desirable to study the analogous problem to $(0.1)$ on a bounded interval and the way a solution of the latter converges to a solution of (0.1). For $a>0$, we let $I_{a}=[-a,+a]$ and we consider the problem : 
$(1.25) \quad\left\{\begin{array}{l}\text { To find } u, v \in \mathscr{C}^{2}\left(I_{a}, \mathbb{R}\right) \text { and } c \geq 0 \text { satisfying: } \\ -u^{\prime \prime}+c u^{\prime}=f(u) v \text { and }-\lambda v^{\prime \prime}+c v^{\prime}=-f(u) v \text { on } I_{a}, \\ -u^{\prime}(-a)+c u(-a)=0, \quad u(0)=z_{0}, \quad u(a)=1, \\ -\lambda v^{\prime}(-a)+c v(-a)=c, \quad v(a)=0,\end{array}\right.$

for some fixed $\left.z_{0} \in\right] 0,1[$.

The purpose of this section is to show, under condition (0.4), the following :

Theorem 1.24 : For any $\mathrm{a}>0,(1.25)$ has (at least) a solution $\left(\mathrm{u}_{\mathrm{a}}, \mathrm{v}_{\mathrm{a}}, \mathrm{c}_{\mathrm{a}}\right.$ ). Moreover, there exists a sequence $\left(a_{n}\right)_{n}$ with $\lim a_{n}=+\infty$ with the property that ( $u_{n}, v_{a_{n}}, c_{a_{n}}$ ) converges to a solution $(u, v, c)$ of $(0.1)$ in $\mathscr{C}^{2}(\mathbb{R}) \times \mathscr{C}^{2}(\mathbb{R}) \times \mathbb{R}$; where $u_{a_{n}}$ (resp $v_{a_{n}}$ ) is extended by $u_{a_{n}}(x)=1 \quad \forall x \geq a_{n}$ and $u_{a_{n}}(x)=u_{a_{n}}\left(-a_{n}\right.$ ) $\forall x \leq-a_{n}\left(\operatorname{resp} v_{a_{n}}(x)=0 \quad \forall x \geq a_{n}\right.$ and $\left.\left.v_{a_{n}}(x)=v_{a_{n}}\left(-a_{n}\right) \quad \forall x \leq-a_{n}\right)\right)$.

Proof : Berestycki, Nicolaenko and Scheurer [1] considered Problem (1.25) in the case of an ignition temperature and their work, which relies on a topological degree argument, can be partly applied here.

Let $x=\mathscr{C}^{1}\left(I_{a}, \mathbb{R}\right) \times \mathscr{C}^{1}\left(I_{a}, \mathbb{R}\right) x \mathbb{R}$. For any $\tau \in[0,1]$, let $L_{\tau}: x \rightarrow X$

be defined by $L_{\tau}((u, v, c))=\left(U, V, c-u(0)+z_{o}\right)$, where $(U, V)$ is the unique solution of the following linear system :

$$
\left\{\begin{array}{l}
-U^{\prime \prime}+c U^{\prime}=\tau f(u) v \text { and }-\lambda V^{\prime \prime}+c V^{\prime}=-\tau f(u) v \text { on } I_{a}, \\
-U^{\prime}(-a)+c U(-a)=0, \quad U(a)=1 \\
-\lambda V^{\prime}(-a)+c V(-a)=c, \quad V(a)=0
\end{array}\right.
$$

If there exist some $\bar{c}>0$ and $R>0$ with the property that $(0,0,0) \notin\left(I-L_{\tau}\right)(\partial \Omega)$

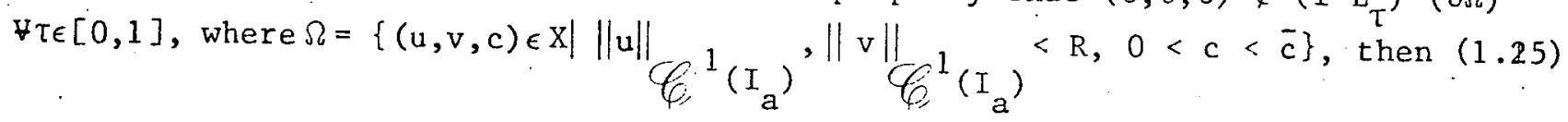
has a solution (see $[1]$ ). 
In the next two lemmae, we derive the a priori estimates which enable to construct such an open set $\Omega$, and next to pass to the limit as $a \rightarrow+\infty$.

Lemma 1.25: Let $\left(u_{a}, v_{a}, c_{a}\right)$ be a solution of (1.25): Then : $c_{a}>0 ; u_{a}>0$ on $I_{a}, 0<u_{a}^{\prime} \leq c_{a}$ and $-\frac{c_{a}}{\lambda} \leq v_{a}^{\prime}<0$ on $I_{a}$ ? $\mathrm{u}_{\mathrm{a}}$ and $\mathrm{v}_{\mathrm{a}}$ satisfy the relations (1.7), (1.8) and (1.9) on $\mathrm{I}_{\mathrm{a}}$.

Proof : See previous lemmae or Berestycki, Nicolaenko and Scheurer [1].

Lemma 1.26: For any $a>0$, let $\left(u_{a}, v_{a}, c_{a}\right)$ be a solution of (1.25).

Then :

$$
c_{a} \geq \sqrt{2 \Lambda \int_{z_{0}}^{1} f(s)(1-s) d s}
$$

(ii) Let $a \geq a_{0}>0$. Then $c_{a} \leq \sup \left(2 \sqrt{\sigma \Lambda^{\prime}}, \frac{2}{a_{0}} \log \frac{1}{z_{0}}\right)$, where $\sigma$ is defined by $(1.1)$.

Proof: The proof of (i) is similar to Lemma 1.5 and is omitted. Let us check (ii). Let $a \geq a_{0}>0$. Then either $c_{a}<2 \sqrt{\sigma \Lambda^{\prime}}$, or $c_{a} \geq 2 \sqrt{\sigma \Lambda^{\prime}}$.

Suppose the latter case occurs. Let $h_{a} \in \mathcal{C}{ }^{1}\left(\left[u_{a}(-a), 1\right]\right)$ be defined by $h_{a}(s)=u_{a}^{\prime}\left(u_{a}^{-1}(s)\right)$. Then, $\forall s \in\left[u_{a}(-a), 1\right], h_{a}^{\prime}(s) \geq c_{a}-\frac{\Lambda^{\prime} \sigma s}{h_{a}(s)}$. Now, if we set $r_{a}=\frac{1}{2}\left(c_{a}+\sqrt{c_{a}^{2}-4 \sigma \Lambda^{\prime}}\right)$, the function $v_{a}(s)=r_{a} s$ satisfies $\frac{d}{d s} v_{a}(s)=c_{a}-\frac{\sigma \Lambda^{\prime} s}{v_{a}(s)}$. Thus, since $v_{a}\left(u_{a}(-a)\right)<h_{a}\left(u_{a}(-a)\right)$, we obtain $v_{a}<h_{a}$ on $\left[u_{a}(-a), 1\right]$, that is $\forall x_{\in}[-a,+a] \quad u_{a}^{\prime}(x)>r_{a} u_{a}(x)$. Therefore, since $u_{a}>0$ on $I_{a}$, one has $: \log \frac{u_{a}(a)}{u_{a}(0)}>r_{a} a \geq r_{a} a_{0}$. This yields $c_{a} \leq \frac{2}{a_{0}} \log \frac{1}{z_{0}}$. Thus, in any case, we have : $c_{a} \leq \sup \left(2 \sqrt{\sigma \Lambda^{\prime}}, \frac{2}{a_{0}} \log \frac{1}{z_{0}}\right)$.

It is straightforward to deduce from these two lemmae the existence of a solution $\left(u_{a}, v_{a}, c_{a}\right)$ of $(1.25)$, for any $a>0$. Moreover since we glvain iniori estimates independent of $a \geq a_{0}$, it is possible to pass to the limit for $\left(u_{a}, v_{n}, c_{\Omega}\right)$ as $a++\infty$ (at least for a sequence) and the nhtnind 1 imit is a solution of $(0.1)$. The proot of Theorem 1.24 is thereby complete. 
Remark 1.27: (1.25) is the analogous problem to $(0.1)$ on a bounded interval but it behaves quite differently. Indeed if $(u, v, c)$ is a solution of (1.25), then c is bounded. In the scalar case $(\lambda=1)$ there is one and only one solution to the problem (1.25) (see[7]). But, on the contrary, the problem (0.1) has a solution of the form $(u, v, c)$ for any large enough $c$. This difference of behaviour does not occur when dealing with the model with ignition temperature (see [1]). 
2. CASE OF THE LEWIS NUMBER LARGER THAN 1.

In this section, we assume that $\lambda$ (which represents the inverse of the Lewis number) satisfies

(2.1) $0<\lambda<1$,

and that $f$ satisfies hypothesis $(0.4)$ and

(2.2) $f$ is of class $C^{1}$ in a neighbourhood of 1 .

Under these assumptions, we complete in Section 2.2, results of Section 1 , namely the study of the solutions of $(0.1)$ and the comprison with the model with ignition temperature. This is possible thanks to a monotony lemma that we first prove in Section 2.1 . Lastly, in Section 2.3, we investigate the high activation energy limits.

\section{2.l Monotony lemma.}

This lemma is an additional result to those of section 1.1. Let some $\left.z_{o} \epsilon\right] 0,1\left[\right.$ be $f$ ixed. In section 1.1 , we associated to any $c>0$ a triplet $\left(u_{c}, v_{c}, h_{c}\right)$ in the following manner :

- $\left(u_{c}, v_{c}\right)$ is the unique couple satisfying (1.13) (see lemma 1.12), . $h_{c}$ is then defined by $(1.16)$.

In the sequel, a key-role will be played by the following proposition :

Proposition 2.1 (Monotony lemma) : Under conditions (0.4), (2.1), (2.2) and $0<c_{1}<c_{2}$, one has $\left.h_{c_{2}}(s)<h_{c_{1}}(s) \quad \forall s \in\right] 0,1[$.

Remark 2.2 : Proposition 2.1 extends a result previously known in the scalar case (see Johnson[5], Marion [7]).

Corollary 2.3 : Under conditions (0.4), (2.1), (2.2) and $0<c_{1}<c_{2}$, one has $u_{c_{2}}(x)>u_{c_{1}}(x) \quad \forall x \cdot<0$ and $u_{c_{2}}(x)<u_{c_{1}}(x) \quad \forall x>0$.

Proof of Proposition 2.1 : We remark that, since $\lambda<1$, one has, from $(1.2), \Lambda=1$ and $\Lambda^{\prime}=\frac{1}{\lambda}$. Thus (1.9) yields $\forall c>0 \quad u_{c}+v_{c}-1>0$ on $\mathbb{R}$ (it is straightforward to verify the strict inequality)= 
For any c > $>$, let us define $1_{c} \mathscr{C}([0,1]) \cap \mathscr{C}^{1}(] 0,1[)$ by :

$$
\begin{cases}\forall s \in] 0,1[ & 1_{c}(s)=\left(u_{c}+v_{c}-1\right)\left(u_{c}^{-1}(s)\right)=s+v_{c}\left(u_{c}^{-1}(s)\right)-1, \\ 1_{c}(1)=0, & 1_{c}(0)=v_{c}\left(u_{c}^{-1}(0)\right)-1 .\end{cases}
$$

Hence, $\left(h_{c}, 1_{c}\right)$ satisfies the differential system :

$$
\left\{\begin{array}{l}
\frac{d}{d s} h_{c}(s)=c-\frac{f(s)(1-s)}{h_{c}(s)}-\frac{f(s) 1_{c}(s)}{h_{c}(s)}, \\
\forall s \in] 0,1[, \\
\frac{d}{d s} 1_{c}(s)=1-\frac{1}{\lambda}+\frac{c}{\lambda} \frac{1_{c}(s)}{h_{c}(s)} .
\end{array}\right.
$$

Lemma 2:4: For any $c>0, h_{c}$ and $I_{c}$ are differentiable at 1. Moreover:

$$
\text { (2.4) } \quad 1_{c}^{\prime}(1)=\left(1-\frac{1}{\lambda}\right)\left(1+\frac{c}{f(1)} \quad h_{c}^{\prime}(1)\right) \text {. }
$$

Proof : Let us denote by $u=u_{c}, v=v_{c}, h=h_{c}$ and $l=1_{c}$. If we suppose that $h$ and $l$ are differentiable at 1 , then we deduce easily from the differential system for $(h, 1)$ that $h^{\prime}(1)$ and $l^{\prime}(1)$ are given by $(2.4)$ and $(2.5)$.

Thus the main point is to prove the differentiability of $h$ and 1 at 1 .

To prove the differentiability of $h$, we consider the dynamical system that we introduced in Lemma 1.12 and we again apply the Stable Manifold Theorem. Indeed, recall that, if we denote by $w=-\lambda v^{\prime}+c v$, then $X=(u, v, w)$ satisfies (1.18), where the vector field $F$ is defined by (1.17). We saw that the derivative $\operatorname{DF}((1,0,0))$ of $F$ at $(1,0,0)$ has two $>0$ eigenvalues and one $<0$ eigenvalue. Moreover, the eigenspace $\mathrm{E}^{-}$associated to the $<0$ eigenvalue'is spanned by the vector:

$$
\left(a_{1}, a_{2}, a_{3}\right)=\left(\frac{2 \lambda}{-\sqrt{c^{2}+4 \lambda f(1)}-2 \lambda c+c}, \frac{2}{c+\sqrt{c^{2}+4 \lambda f(1)}}, 1\right)
$$

The stable Manifold Theorem asserts that the tangent space at $(1,0,0)$ of the local 
stable manifold is $E^{-}$. Hence, one has $\lim _{x \rightarrow+\infty} \frac{w(x)}{u(x)-1}=\frac{a_{3}}{a_{1}}$; since $u^{\prime}=c(u-1)+w$,

this leads to $\lim _{x \rightarrow+\infty} \frac{u^{\prime}(x)}{u(x)-1}=\frac{a_{3}}{a_{1}}+c$. Thus $h$ is differentiable at 1 and moreover $(2.3)$ is showed.

It remains to prove the differentiability of 1 at 1 . Let us argue by contradiction and let $m \in]_{\frac{1 i m}{s+1}} \frac{1(s)}{s-1}, \overline{1 i m}_{s \rightarrow 1} \frac{1(s)}{s-1}[$. Then, from the equation satisfied by 1 , one deduces $m=\left(1-\frac{1}{\lambda}\right)\left\{1-\frac{c}{\lambda h^{\prime}(1)}\right\}^{-1}$, which is impossible. Hence 1 is differentiable at 1 and one sees easily that $I^{\prime}(1)$ is equal to the above expression for $m$; combining this expression and (2.3), one obtains (2.4).

End of the proof o E Proposition 2.1 : Let $0<c_{1}<c_{2}$ and, $F i=1,2$, let us denote by $h_{i}=h_{c_{i}}$ and $l_{i}=l_{c_{i}}$.

From Lemme 2.4, we know that $h_{1}^{\prime}(1)<h_{2}^{\prime}(1)$ and $I_{1}^{\prime}(1)<I_{2}^{\prime}(1)$. Thus, since $h_{i}>0$ and $1_{1}>0$ on $] 0,1\left[\right.$, there exists some $\left.s_{0} \epsilon\right] 0,1[$ with the property that :

(2.5) $h_{1}>h_{2}$ and $l_{1}>I_{2}$ on $]_{0}, 1[$.

Let us argue by contradiction and prove that (2.5) yields $h_{1}>h_{2}$ and $l_{1}>1_{2}$ on $] 0,1[$. Indeed, if not, there exists some $\alpha=] 0,1$ s such that $h_{1}>h_{2}$ and $I_{1}>I_{2}$ on $] a, 1[$, and, $b_{1}(\alpha)=h_{2}(\alpha)$, or $l_{1}(\alpha)=l_{2}(\alpha)$. In the first case, taking the difference of the equations for $h_{1}$ and $h_{2}$, one has :

$h_{2}^{\prime}(\alpha)-h_{1}^{\prime}(\alpha)=c_{2}-c_{1}-\frac{f(\alpha)}{h_{2}(\alpha)}\left(1_{2}(\alpha)-1_{1}(\alpha)\right)>0$

which is impossible. In the same way, in the latter case, we obtain a contradiction since :

$I_{2}^{\prime}(\alpha)-I_{1}^{\prime}(\alpha)=\frac{1}{\lambda} I_{2}(\alpha) \cdot\left(\frac{c_{2}}{h_{2}(\alpha)}-\frac{c_{1}}{h_{1}(\alpha)}\right)>0$

This completes the proof of Proposition 2.1 .

Remark 2.5 : The assumption $\lambda<1$ is crucial for this last argument. Notice that, if $\lambda>1$, then $1_{i}<0$. 
Remark 2.6 :'This type of monotony results has been known since the formal works of Kane1'[6], but it is the first time, as far as we know, that a rigorous proof is given.

\subsection{Applications}

\subsection{1 - Determination of the set of solutions of $(0.1)$}

The following result refines that of Theorem 1.1 :

Theorem 2.7 : Under conditions $(0.4),(2.1)$ and (2.2):

(i) There exists some $c_{0}>0$ such that $(0.1)$ has a solution $(u, v, c)$ if and only if $c \geq c_{0}$.

(ii) For any $\mathrm{c} \geq \mathrm{c}_{0}$, there exists an unique couple $(\mathrm{u}, \mathrm{v})$ (up to a translation of the origin) such that $(u, v, c)$ is solution of $(0.1)$.

(iii) Mareover,

$$
\sqrt{2 \int_{0}^{1} f(s)(1-s) d s}<c_{0} \leq 2 \sqrt{\frac{1}{\lambda} \sup _{x \in] 0,1\left[\frac{f(x)(1-x)}{x}\right.}} .
$$

Proof : Let us set $\mathscr{C}=\{c ;(0.1)$ has a solution $(u, v, c)\}$. From Theorem 1.1, we know that there exist $0<\alpha \leq \beta$ such that $[\beta+\infty[\mathcal{C}$ and $\forall x \in \mathscr{C}$ one has $x \geq \alpha$. We deduce from Corollary 2.3 (see Remark 1.10) that $\varnothing$ is an interval. Hence there exists some $c_{0}>0$ such that $\mathscr{C}=\left(c_{0},+\infty\left[\right.\right.$ and it is easily seen that $c_{0} \epsilon \varnothing$, by passing to the limit as $c \rightarrow c_{0}, c>c_{0}$, for the solutions of $(0.1)$; this concludes the proof of (i). (ii) and (iii) are consequences of Theorem 1.1.

Remark 2.8 : Theorem 2.7 generalizes a result previously known in the scalar case (see Fife [4], Johnson [5], Marion [7], Uchi yama [9]).

\subsubsection{Relationship with the model with ignition temperature.}

Before investigating this relationship, let us consider the model with ignition tem perature. Under condition (0.2), Berestycki, Nicolaenko and Scheurer proved, for any $\lambda>0$, the existence of a solution of $(0.1)$. Thanks to the monotony lemma, we shall derive here the uniqueness of such a solution in the case $\lambda<1$. More precisely, the following theorem holds : 
Theorem 2.9: We assume that condition (0.2) holds with 6 Lipschitz continuous on $[\theta, 1]$ and of class $C, 1$ in a neighbourhood of 1 . Then, if $0<\lambda<1$, there exists one and only one solution of (0.1) (up to a translation of the origin).

Proof : To prove the uniqueness, we argue by contradiction. Let $\left(u_{1}, v_{1}, c_{1}\right)$ and $\left(u_{2}, v_{2}, c_{2}\right)$ be two different solutions of $(0.1)$, with $\forall i=1,2 u_{i}(0)=\theta$. Then according to the stable Manifold Theorem (see the proof of Lemma 1.12), if $c_{1}=c_{2}$, we would have $u_{1}=u_{2}$ and $v_{1}=v_{2}$. Thus we have necessarily $c_{1} \neq c_{2}$.

Suppose $c_{1}<c_{2}$. $\forall i=1,2$, let us associate $h_{i}$ to $\left(u_{i}, v_{i}, c_{i}\right)$ by $(1.20)$. Then the arguments of the proof of Proposition 2.1 show that $h_{2}<h_{1}$ on $[\theta, 1[$. In particular $h_{2}(\theta)<h_{1}(\theta)$, which is impossible since $h_{i}(\theta)=c_{i} \theta$.

Remark 2.10 : Theorem 2.7 and Theoren 2.9 emphasize the great difference in the behaviour of the solutions of the two models. Nevertheless the solutions of these two models can be closely connected. It is the purpose of the next theorem.

$\forall \theta \in] J, 1\left[\right.$, let us denote by $\left(u_{\theta}, v_{\theta}, c_{\theta}\right)$ the unique solution of (1.19). The following theorem improves Theorem 1.14 :

Theorem 2.11 : Under conditions $(0.4),(2.1)$ and $(2.2)$ :

(i) The function: $] 0,1\left[\rightarrow \mathbb{R}^{+*}\right.$. is strictly increasing.

(ii) $\left(u_{\theta}, v_{\theta}, c_{\theta}\right)$ converges to $\left(u_{0}, v_{0}, c_{0}\right)$ in $\mathscr{c}_{\theta}^{2}(\mathbb{R}) \times f^{2}(\mathbb{R}) x \mathbb{R}$, as $\theta \rightarrow 0$, where $c_{0}$ is defined at Theorem 2.6 (i) and $\left(u_{0}, v_{0}, c_{0}\right)$ is the unique corresponding solution of $(0.1)$ with $u_{0}(0)=z_{0}$.

Proof : The monotony lemma enables us to prove this theorem by arguments similar to those of the scalar case; the proof is omitted (see [5,7] and the a-priori estimates are obtained in Theorem 1.14). 


\subsection{Further remarks on the asymptotic analysis.}

We assume that $f$ depends on a parameter $\varepsilon>0\left(f=f_{\varepsilon}\right)$ and that the family $\left(f_{\varepsilon}{ }^{\ddagger} \varepsilon\right.$ satisfies conditions $(1.21),(1.22),(1.23)$ and $(1.24)$. Let some $\left.z_{0} \in\right] 0,1[$ be fixed. For any $\varepsilon>0$, the problem :

(2.6) $\left\{\begin{array}{l}-\mathrm{u}^{\prime \prime}+\mathrm{cu} \mathrm{u}^{\prime}=\mathrm{f}_{\varepsilon}(\mathrm{u}) \mathrm{v} \\ -\lambda \mathrm{v}^{\prime \prime}+\mathrm{c} \mathrm{v}^{\prime}=-\mathrm{f}_{\varepsilon}(\mathrm{u}) \mathrm{v} \\ \mathrm{u}(-\infty)=0, \quad \mathrm{u}(0)=z_{0}, \quad \mathrm{u}(+\infty)=1, \\ \mathrm{v}(-\infty)=1, \quad \mathrm{v}(+\infty)=0 .\end{array}\right.$

has got infinitely many solutions (for $c \geq c_{0, \varepsilon}>0$ ). Moreover, $\forall c \geq c_{0, \varepsilon}$ the corresponding solution of (2.6) is unique. In this section, we are concerned with the asymptotic behaviour of these solutions, as $\varepsilon \rightarrow 0$.

First, let us deal with the solution corresponding to. $c_{0, \varepsilon}$, that we denote by $\left(u_{o, \varepsilon} ; v_{o, \varepsilon}, c_{o, \varepsilon}\right)$ :

Proposition 2.12 : Under conditions (1.21), (1.22), (1.23) and (1.24):

(i) $\overline{\lim }_{\varepsilon \rightarrow 0} c_{o, \varepsilon} \leq \sqrt{2 \Lambda^{\prime} \mathrm{m}}$, and $\frac{\lim }{\varepsilon \rightarrow 0} c_{o, \varepsilon} \geq \sqrt{2 \Lambda \mathrm{m}}$.

(ii) Let $\left(\varepsilon_{n}\right)$ be a sequence such that $1 \mathrm{im} \varepsilon_{\mathrm{n}}=0$ and $\lim c_{0, \varepsilon_{\mathrm{n}}}=c_{0}$. Then $\left(\mathrm{u}_{0, \varepsilon_{\mathrm{u}}}, \mathrm{v}_{0, \varepsilon_{\mathrm{n}}}\right)$ converges to $\left(u_{0}, v_{0}\right)$ in $\ell_{0}^{0}(\mathbb{R}) \times \mathscr{C}^{0}(\mathbb{R})$, as $n \rightarrow+\infty$, where $\left(u_{0}, v_{0}\right)$ is uniquely determined by:

$$
\left\{\begin{array}{c}
-u_{0}^{\prime \prime}+c_{0}^{u_{0}^{\prime}=}=c_{0} \delta{ }_{x=x_{0}}, \\
-\lambda v_{0}^{\prime \prime}+c_{0} v_{0}^{\prime}=-c_{0}^{\delta}, x_{x=x_{0}}, \\
u_{0}(-\infty)=0, \quad u_{0}(+\infty)=1, \\
v_{0}(-\infty)=1, \quad v_{0}(+\infty)=0
\end{array}\right.
$$

and $\mathrm{x}_{0}$ is determined by the condition $\mathrm{u}_{0}(0)=z_{0}$. 
Proof : It is a straighforward consequence of Theorem 2.11 and Theorem 1.19 .

Now, since $\frac{\lim }{\varepsilon \rightarrow 0} c_{0, \varepsilon} \leq \sqrt{2 \Lambda^{\prime} \mathrm{m}}$, for any $c>\sqrt{2 \Lambda^{\prime} \mathrm{m}}$, we can introduce the solution $\left(u_{c, \varepsilon}, v_{c, \varepsilon}, c\right)$ of $(2.6)$ (defined for $\left.\varepsilon \leq \varepsilon_{0}\right)$ and we can investigate the asymptotic

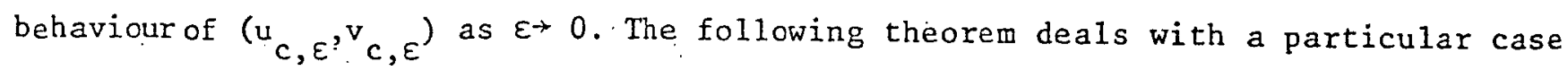
where the limit of $\left(u_{c, \varepsilon}, v_{\varepsilon ; \varepsilon}\right)$, as $\varepsilon \rightarrow 0$, is entirely determined; in the general case, we obtain results only for sequences (see Remark 2.15 below).

Theorem 2.13 : Una'er conditions (1.21), (1.22), (1.23) and (1.24), let some c> $\sqrt{2 \Lambda^{\prime} m}$ be fixed and let us denote by $\left(u_{c, \varepsilon}, v_{c, \varepsilon}, c\right)$ the corresponding solution of (2.6) lafined for $\left.\varepsilon \leq \varepsilon_{0}\right)$. Then, under the additional condition $\left.z_{0} \epsilon\right] 0, \frac{c-\sqrt{2 m \Lambda^{\prime}}}{c}[$, $\left(u_{c, \varepsilon}, v_{c, \varepsilon}\right)$ converges to $(u, v)$ in $\mathscr{C}_{10 c}^{0}(\mathbb{R}) \times C_{10 c}^{0}(\mathbb{R})$, as $\varepsilon \rightarrow 0$, where $u$ (resp v) is the function : $x \rightarrow z_{0}$ (resp : $x \rightarrow 1-z_{0}$ ).

Proof : $\forall \varepsilon \leq \varepsilon_{0}$, one has $u_{c, \varepsilon}^{\prime}\left(z_{0}\right)=h_{c, \varepsilon}\left(z_{0}\right)$ where $h_{c, \varepsilon}$ is the function associated to $\left(u_{c, \varepsilon}, v_{c, \varepsilon}, c\right)$ by $(1.16)$. Theorem 2.13 is then an easy consequence of the next lemma :

Lemma 2.14: Under conditions (1.21), (1.22), (1.23) and (1.24), one has
(i) $\forall s \in] 0,1\left[\quad \frac{\lim }{\frac{\varepsilon+0}{1 m}} h_{c, \varepsilon}(s) \geq \sup (0, c s-c+\sqrt{2 \Lambda m})\right.$,
(ii) $\forall s \in] 0,1\left[\quad \lim _{\varepsilon \rightarrow 0} h_{c, \varepsilon}(s) \leq \sup \left(0, c s-c+\sqrt{2 \Lambda^{\prime} m}\right)\right.$.

Proof of Lemma 2.14 : Let any $s \in] 0,1\left[\right.$ be fixed. $\forall \varepsilon\left(\leq \varepsilon_{0}\right)$, let us denote by ( $\left.{ }_{\varepsilon}, v_{\varepsilon}, c\right)$ the solution of (2.6) with $u_{\varepsilon}(0)=s$ ( $u_{\varepsilon}$ differs from $u_{c}, \varepsilon$ by a translation of the $x$-axis). Let us write $(1.10),(1.11)$ and $(1.12)$ (for $\left(u_{\varepsilon}, v_{\varepsilon}, c\right)$ and $\left.f_{\varepsilon}\right)$ with $a=0$ and $b=+\infty$; by an argument similar to the one of lemma 1.5, we obtain : 
$\frac{1}{2}\left(u_{\varepsilon}^{\prime}(0)\right)^{2}+c(1-s) u_{\varepsilon}^{\prime}(0)+\frac{c^{2}}{2}(1-s)^{2}-\Lambda \int_{s}^{1} f_{\varepsilon}(\tau)(1-\tau) d \tau \geq 0$

which yields $u_{\varepsilon}^{\prime}(0) \geq-c(1-s)+\sqrt{2 \Lambda \int_{s}^{1}} f_{\varepsilon}(\tau)(1-\tau) d \tau$.

It is now straightforward to complete the proof of (i), since $h_{c, \varepsilon}(s)=u_{\varepsilon}^{\prime}(0)$.

From Lemma 1.23, we know that the application $h_{o, \varepsilon}$ associated to $\left(u_{o, \varepsilon}, v_{o, \varepsilon}, c_{o, \varepsilon}\right.$ ) by (1.16) satisfies :

$\forall s \in\left[0,1\left[\lim _{\varepsilon \rightarrow 0}\left(h_{\delta, \varepsilon}(s)-c_{o, \varepsilon} s\right)=0\right.\right.$.

In particular, $\overline{\lim }_{\varepsilon \rightarrow 0} h_{o, \varepsilon}(s) \leq \overline{\lim }_{\varepsilon \rightarrow 0} c_{o, \varepsilon} s \leq \sqrt{2 \Lambda^{\prime} m} s$.

From the monotony lemma, we deduce $h_{c, \varepsilon} \leq h_{o, \varepsilon}$ on $] 0,1[$.

Combining these inequalities, we obtain

(2.7) $\forall s \in] 0,1\left[\overline{\lim }_{\varepsilon \rightarrow 0} \cdot h_{c, \varepsilon}(s) \leq \sqrt{2 \Lambda^{\prime} \mathrm{m}} \mathrm{s}\right.$.

(ii) can then be deduced from (2.7) by an argument similar to the one of the scalar case $[7]$.

End of the proof of Theorem 2.13 : In the case $\left.\left.z_{0} \epsilon\right] 0, \frac{c-\sqrt{2 m \Lambda^{\prime}}}{c}\right]$, Lemma 2.14 yields $\lim _{\varepsilon \rightarrow 0} u_{c, \varepsilon}^{\prime}(0)=0$, and the proof is now easily completed.

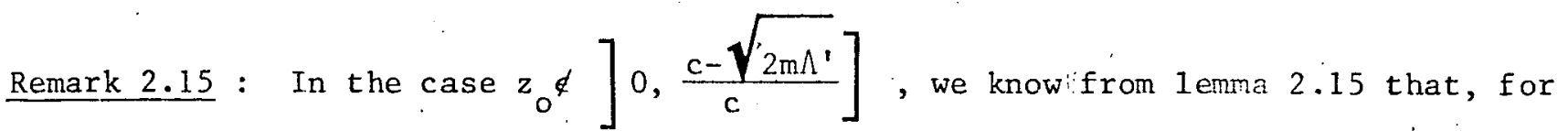
any sequence $\left(\varepsilon_{\mathrm{n}}\right)$ with $\lim \varepsilon_{\mathrm{n}}=0$ such that there exists $(u, v)=1$ im $\left(u_{c, \varepsilon_{n}}, v_{c, \varepsilon_{n}}\right)$, there are two possibilities for $(u, v)$ :

- if $\lim u_{c, \varepsilon_{n}}^{\prime}(0)=0,(u, v)$ is the couple defined at Theorem 2.13,

- if $\lim _{\mathrm{c}, \varepsilon_{\mathrm{n}}}^{\prime}(0)=\delta>0$, (u,v) is uniquely determined by :

$\begin{cases}-u^{\prime}+c u=-\delta+c z_{o} & \text { on }]-\infty, \bar{x}[, \\ -\lambda v^{\prime}+c v=c\left(1-z_{c}\right)+\delta & \text { on }]-\infty, \bar{x}[, \\ u \equiv 1 \text { and } v=0 \text { on } \cdot[\bar{x}+\infty[, & \end{cases}$

and $\bar{x}$ is uniquely determined by the condifin $1(0)=?$. 
Moreover, one has :

$\sup \left(0, c z_{0}-c+\sqrt{2 \Lambda m}\right) \leq \delta \leq \sup \left(0, c z_{0}-c+\sqrt{2 \Lambda^{\prime} m}\right)$

In any case, one has $u(-\infty) \neq 0$ and $v(-\infty) \neq 1:$ the boundary conditions at $-\infty$ are no more satisfied; this result generalizes the one obtained in the scalar case (we proved in [7] that the asymptotic behaviour of the solutions which do not correspond to the minimal $c$ is not physical : at the limit $\varepsilon=0$, one does not find any "fresh gas" as one goes towards $-\infty$ ). 


\section{REFERENCES}

[1] BERESTYCKI, H., NICOLAENKO, B. and SCHEURER, B., "Traveling wave solutions to combustion models and their singular limits", to appear.

[2] BUCKMASTER, J., and LUDFORD, G.S.S., "The laminar flame theory", Cambridge University Press, (1982).

[3] CHILLINGWORTH, D.R.J., "Differential topology with a view to applications", Pitman, London, (1976).

[4] FIFE, P.C., "Mathematical aspects of reacting and diffusing systems", Lecture Notes in Biomathematics 28, Springer Verlag, (1979).

[5] JOHNSON, W.E., "On a first order boundary value problem from laminar flame theory", Arch. Rat. Mech. Ana1., 13, 1963, 46-54.

[6] KANEL', YA. I., "On steady state solutions to systems of equations arising in combustion theory", Dok1. Akad. Nauk. U.S.S.R., 149, No. 2, 367-369 (1963).

[7] MARION, M., "Etude mathématique d'un modèle de flamme laminaire sans température d'ignition : I - Cas scalaire, "Annales de la Faculté des Sciences de Toulouse, to appear.

[8] RABINOWITZ, P., "Theorie du degré topologique et applications à des problèmes aux limites non linéaires", Lecture Notes 75010, University of Paris, (1975).

[9] UCHIYAMA, K., "The behaviour of some non linear diffusion equations for large time", J. Math. Kyoto Univ., 18, (1978), 453-508.

[10] WILLIAMS, F., "Combustion theory", Addison-Wesley (1963). 
